\title{
An Unconditionally Stable, Energy-Momentum Consistent Implementation of the Material-Point Method
}

\section{Authors}

E. Love

The University of New Mexico

Department of Mathematics and Statistics

D. L. Sulsky

The University of New Mexico

Department of Mathematics and Statistics 


\section{Disclaimer}

The Center for High Performance Computing (HPC@UNM)

provides a focus for high performance computing and communication at the University of New Mexico (UNM). HPC@UNM is committed to innovative research in computational and computer science with emphasis on both algorithm development and application. As part of this commitment, HPC@UNM sponsors this technical report series. The technical reports are subject to internal review by HPC@UNM. However, the material, as presented, does not necessarily reflect any position of HPC@UNM. Further, neither UNM, nor the HPC, makes any warranty or assumes any legal liability or responsibility for the accuracy, completeness, or usefulness of any information contained in this report. 


\title{
An Unconditionally Stable, Energy-Momentum Consistent Implementation of the Material-Point Method
}

\author{
E. Love and D. L. Sulsky* \\ Department of Mathematics and Statistics, 415 Humanities Building, \\ MSC03 2150, University of New Mexico, Albuquerque, NM 87131-0001
}

September 9, 2004

\begin{abstract}
Conservation of linear and angular momenta, and conservation of energy are examined for the material-point method (MPM). It is shown that MPM can be formulated with implicit energy and momentum conserving mesh dynamics for hyperelastic materials. With a consistent mass matrix the resulting overall numerical method preserves the conservative properties of the mesh solution. Energy dissipation and angular momentum errors are also quantified for a lumped mass formulation. Properties of the method are illustrated in numerical examples.
\end{abstract}

Keywords: Material-Point Method (MPM), Energy Conservation, Momentum Conservation, Unconditional Stability, Finite Deformation Elasticity

\section{Introduction}

The material-point method (MPM) is a general numerical tool for the mechanical analysis of continuous media [42], [44], [45]. This method utilizes two discretizations to solve the continuum equations describing the motion and deformation of fluids and solids. A Lagrangian description of the material under consideration is given by a set of unconnected material points that are tracked throughout the deformation. A background computational grid is used to calculate the interactions among the Lagrangian points through the solution of the momentum equation. A mapping between the two discretizations is performed at each step in the loading process. The constitutive equations are solved at each material point so that material models with history dependence are implemented easily. The method has proven to be particularly useful for problems displaying the large deformation that occurs with inelastic behavior of solids. It is also suited to problems with complicated geometry and multiple materials in contact.

*corresponding author. email: sulsky@math.unm.edu 
The material-point description is able to represent the geometry of a material and its deformation without mesh tangling, and can advect material properties without numerical diffusion or artificial mixing. Solving the momentum equation using this unconnected set of points would involve determining neighbors and computing nearest-neighbor interactions. In order to keep the computational cost linear in the number of material points, information from the points is transferred to a background grid where the momentum equation is solved. The background grid is under user control and can be chosen for computational convenience. In particular, the grid does not have to conform to the boundary of the solid body, so mesh generation can be greatly simplified. The solution technique on the background grid can be based either on finite differences or finite elements. This paper exploits developments in finite element analysis to construct an energy and momentum conserving, unconditionally stable method for hyperelastic solids.

Section 2 of this paper presents the continuum equations and reviews their conservation properties. These properties are well known and appear in many references. The derivations are repeated in this paper so that a direct comparison with the discrete equations can be made. The goal is to replicate these continuum properties in the discrete equations. Section 3 reviews the MPM computational cycle and examines conservation properties of the discretization. The computational cycle consists of four steps: (1) project information from material points to a background grid, (2) solve equations of motion on the grid, (3) transfer information from the grid solution to update the material points, and (4) regrid, if desired. In essence, it is shown that the mapping from material points to grid, and the mapping from grid to material points preserve conservation properties of the grid solution, if the consistent mass matrix is used. With a lumped mass matrix, energy is dissipated and angular momentum is no longer exactly conserved.

Since the material-point kinematics are the same as the particle updates in the particlein-cell method, FLIP, the discussion of conservation properties of the material-point-to-grid projection, and of the material-point update closely follows [11], [12], [13] and [16]. Since FLIP is a hydrodynamics code, the principal difference between MPM and FLIP is in the treatment of the material constitutive model. Newtonian fluids are not history dependent, so there is no need to carry stresses or deformation gradients as material-point quantities. Particle data are interpolated to the grid using shape functions, and then differentiated on the grid when necessary. The literature on FLIP also describes the grid solution in terms of finite differences, whereas in this paper the connection to finite elements is emphasized. In order to have a completely conservative method, the Lagrangian grid dynamics must also conserve energy and momentum. For this step we make use of an implicit time integration scheme developed for the finite element method which has the desired properties. Thus, analysis of the grid dynamics follows [21], [40] and [41]. There are some details of the overall analysis in this paper that differ from the references, and pieces that are drawn from several references. Therefore, this paper collects, and hopefully clarifies, conservation properties of MPM and its connection to finite element methods.

Finally, in Section 4, numerical examples are presented that illustrate the properties of this energy and momentum conserving material-point method. Section 5 closes the paper with some concluding remarks and observations. 


\section{The continuum problem}

The goal of this section is to present the continuum equations of motion for a hyperelastic solid body. These equations are well known, and the reader may consult the books [1], [17], [24], [33] or [38] for more information. After introducing the notation, the weak form of the linear momentum equation and the hyperelastic constitutive model are given. Next, conservation of linear momentum, angular momentum and energy are reviewed for the unforced, traction-free Neumann problem. The presentation in this section follows closely that of Section 2 in reference [40].

\subsection{Basic notation}

Let $\Omega_{0} \in \mathbb{R}^{3}$ denote the reference (material) position of a continuum body, with material points labeled $\mathbf{X}$. The set $\Omega_{0}$ is assumed open and bounded, with a smooth boundary $\Gamma_{0}$. Assume the reference boundary $\Gamma_{0}$ is partitioned into disjoint subsets such that $\Gamma_{0}=\overline{\Gamma_{\varphi} \cup \Gamma_{\boldsymbol{\sigma}}}$ and $\Gamma_{\varphi} \bigcap \Gamma_{\sigma}=\emptyset$.

Let the spatial (current) position of the same body be $\Omega \in \mathbb{R}^{3}$, with points labeled $\mathbf{x}$. Assume there exists a smooth mapping, the motion of the body, $\boldsymbol{\varphi}: \Omega_{0} \times[0, \mathrm{~T}] \rightarrow \mathbb{R}^{3}$, such that $\Omega=\boldsymbol{\varphi}\left(\Omega_{0}, \mathrm{t}\right)$ and $\mathbf{x}=\boldsymbol{\varphi}(\mathrm{X}, \mathrm{t})$, where $[0, \mathrm{~T}] \subset \mathbb{R}$ is the the time interval of interest. The deformation gradient of the motion is defined as

$$
\mathbf{F}(\mathrm{X}, \mathrm{t}):=D \varphi=\operatorname{GRAD}_{\mathbf{X}}[\boldsymbol{\varphi}]
$$

Given the above, the set of admissible configurations is then defined as

$$
\mathcal{S}:=\left\{\boldsymbol{\varphi}: \Omega_{0} \times[0, \mathrm{~T}] \rightarrow \mathbb{R}^{3} \mid J(\boldsymbol{\varphi}):=\operatorname{det}[\mathbf{F}]>0 \text { and }\left.\boldsymbol{\varphi}\right|_{\Gamma_{\varphi}}=\bar{\varphi}\right\}
$$

where $\bar{\varphi}: \Gamma_{\boldsymbol{\varphi}} \times[0, \mathrm{~T}] \rightarrow \mathbb{R}^{3}$ is specified on $\Gamma_{\boldsymbol{\varphi}}$. The material velocity of a point $\mathbf{X}$ is defined as $\mathbf{v}:=\dot{\varphi}$, where the superposed dot denotes the material time derivative. Given a reference density $\rho_{0}: \Omega_{0} \rightarrow \mathbb{R}^{+}$, the spatial density is $\rho=J^{-1} \rho_{0}$ and the material momentum is defined as $\boldsymbol{\pi}:=\rho_{0} \mathbf{v}$.

Let $\boldsymbol{\sigma}$ denote the Cauchy stress in the current configuration. The developments in this work critically use the symmetric Piola-Kirchhoff stress $\mathbf{S}$, defined such that

$$
J \boldsymbol{\sigma}=\mathbf{F S F}^{T}
$$

The prescribed traction boundary condition is

$$
(\mathbf{F S}) \mathbf{N}=\overline{\mathbf{T}} \text { on } \Gamma_{\boldsymbol{\sigma}}
$$

where $\mathbf{N}: \Gamma_{0} \rightarrow S^{2}$ is the outward unit normal field to the material boundary $\Gamma_{0}$ and $\overline{\mathbf{T}}: \Gamma_{\boldsymbol{\sigma}} \times[0, \mathrm{~T}] \rightarrow \mathbb{R}^{3}$ is the prescribed nominal traction vector. In addition to these boundary tractions, the body is assumed to be acted upon by a body force (per unit mass) $\mathbf{b}: \Omega_{0} \times$ $[0, \mathrm{~T}] \rightarrow \mathbb{R}^{3}$. 


\subsection{The weak form of balance of linear momentum}

Denote by $\mathcal{W}$ the space of test functions associated with the admissible configurations in $\mathcal{S}$, defined in standard fashion as

$$
\mathcal{W}:=\left\{\delta \varphi: \Omega_{0} \rightarrow \mathbb{R}^{3}|\delta \boldsymbol{\varphi}|_{\Gamma_{\varphi}}=\mathbf{0}\right\}
$$

It is convenient to introduce two inner product notations, $\langle\cdot, \cdot\rangle_{\Omega_{0}}$ and $\langle\cdot, \cdot\rangle_{\Gamma_{0}}$, denoting the $L^{2}$ inner product on $\Omega_{0}$ and $\Gamma_{0}$, respectively, of scalars, vectors or tensors, depending on the context.

With this notation in hand, the weak form of the balance of linear momentum (principle of virtual work in the reference configuration, see e.g., Theorem 2.6-2 in [17]) is: find $\varphi \in \mathcal{S}$ such that

$$
\langle\delta \boldsymbol{\varphi}, \dot{\boldsymbol{\pi}}\rangle_{\Omega_{0}}+\left\langle\mathrm{GRAD}_{\mathbf{X}}[\delta \boldsymbol{\varphi}], \mathbf{F S}\right\rangle_{\Omega_{0}}-\left\langle\delta \boldsymbol{\varphi}, \rho_{0} \mathbf{b}\right\rangle_{\Omega_{0}}-\langle\delta \boldsymbol{\varphi}, \overline{\mathbf{T}}\rangle_{\Gamma_{0}}=0
$$

for all $\delta \varphi \in \mathcal{W}$. Along with the above variational equation, the solution $\varphi$ must also satisfy the initial conditions

$$
\boldsymbol{\varphi}(\mathbf{X}, 0)=\boldsymbol{\varphi}_{0} \text { and } \boldsymbol{\pi}(\mathbf{X}, 0)=\rho_{0} \mathbf{v}_{0} \text { in } \Omega_{0} .
$$

Once a suitable constitutive equation relating $\mathbf{S}$ to the motion $\boldsymbol{\varphi}$ is specified, equations (6) and $(7)$ together define a (generally) nonlinear initial boundary value problem for the motion $\varphi \in \mathcal{S}$ of the body.

\subsection{Constitutive equations}

Definition 2.1. (Reference [37], Part IV, Section 15.3). If the stresses in a body are derivable from a strain-energy function, the material is called hyperelastic.

Denote by $\mathcal{T}_{3 \times 3}$ the space of all rank two tensors. Let $\mathcal{T}_{3 \times 3}^{\text {sym }}$ be the subspace of all symmetric rank two tensors and $\mathcal{T}_{3 \times 3}^{\text {sym+ }}$ be the subset of all symmetric, positive-definite rank two tensors. Then, for a body consisting of a hyperelastic material, at each material point $\mathbf{X} \in \Omega_{0}$ there exists a stored (internal) energy function $W: \mathcal{T}_{3 \times 3}^{\text {sym+ }} \rightarrow \mathbb{R}^{+}$. Without loss of generality, $W$ is assumed to be a function of $\mathbf{C}:=\mathbf{F}^{T} \mathbf{F}$, the right Cauchy-Green strain tensor. This energy function is required to satisfy identically a reduced form of the second law of thermodynamics

$$
\mathcal{D}^{\text {int }}:=\frac{1}{2} \mathbf{S}: \dot{\mathbf{C}}-\dot{W}=0 .
$$

This thermodynamic requirement results in the constitutive equation

$$
\mathbf{S}=\hat{\mathbf{S}}(\mathbf{C}):=2 \frac{\partial W}{\partial \mathbf{C}}(\mathbf{C})
$$

General strain-energy functions, $W$, allow quite general material behavior, including phase transitions and the development of complicated microstructures. The hyperelastic model is sometimes required to satisfy other constitutive inequalities, such as strong ellipticity or polyconvexity. However, these properties are not directly used in this presentation, and there is no need to elaborate upon them. References [1], [17] and [33] provide detailed information on constitutive inequalities. 


\subsection{Conservation properties}

The evolutionary equation (6) possesses a number of important global conservation properties. First, the conserved quantities, linear momentum, angular momentum, and total energy, are defined. Next, it is shown that (6) conserves these quantities under appropriate conditions on the loading and boundary conditions.

Definition 2.2. The total linear momentum $\mathbf{L}$ is defined as

$$
\mathbf{L}:=\int_{\Omega_{0}} \boldsymbol{\pi} \mathrm{d} \Omega_{0} .
$$

Definition 2.3. The total angular momentum $\mathbf{J}$ is defined as

$$
\mathbf{J}:=\int_{\Omega_{0}}(\boldsymbol{\varphi} \times \boldsymbol{\pi}) \mathrm{d} \Omega_{0} .
$$

Definition 2.4. The total kinetic energy $\mathrm{T}$ is defined as

$$
2 \mathrm{~T}:=\left\langle\boldsymbol{\pi}, \rho_{0}^{-1} \boldsymbol{\pi}\right\rangle_{\Omega_{0}}=\langle\boldsymbol{\pi}, \mathbf{v}\rangle_{\Omega_{0}}=\left\langle\rho_{0} \mathbf{v}, \mathbf{v}\right\rangle_{\Omega_{0}} .
$$

Definition 2.5. The total potential energy $\mathrm{V}$ is defined as

$$
\mathrm{V}:=\int_{\Omega_{0}} W(\mathbf{C}) \mathrm{d} \Omega_{0}
$$

Definition 2.6. The total energy $\mathrm{H}$ is defined as

$$
\mathrm{H}:=\mathrm{T}+\mathrm{V}
$$

Theorem 2.1. Let $\Gamma_{\varphi}=\emptyset, \mathbf{b}=\mathbf{0}$ and $\overline{\mathbf{T}}=\mathbf{0}$. Then total linear momentum $\mathbf{L}$, the total angular momentum $\mathbf{J}$, and the total energy $\mathbf{H}$, are constants of the motion.

Proof. This result is well known, and is based on classical arguments. It is included for completeness. The proof is very similar to that of Proposition 2.1 in [2].

1. Linear Momentum. Given that $\Gamma_{\varphi}=\emptyset$, an admissible variation is

$$
\delta \boldsymbol{\varphi}(\mathrm{X})=\boldsymbol{\xi} \in \mathbb{R}^{3} .
$$

Consequently, GRAD $\mathbf{X}[\delta \boldsymbol{\varphi}]=\mathbf{0}$ for this case. Using Definition 2.2 and the weak form (6) with the variation above, and noting that $\mathbf{b}=\mathbf{0}$ and $\overline{\mathbf{T}}=\mathbf{0}$ by assumption, produces

$$
\boldsymbol{\xi} \cdot \frac{\mathrm{d}}{\mathrm{dt}} \mathbf{L}=\langle\boldsymbol{\xi}, \dot{\boldsymbol{\pi}}\rangle_{\Omega_{0}}=-\left\langle\mathrm{GRAD}_{\mathbf{X}}[\boldsymbol{\xi}], \mathbf{F S}\right\rangle_{\Omega_{0}}=0 \quad \forall \boldsymbol{\xi} \in \mathbb{R}^{3}
$$

Therefore, linear momentum is a constant of the motion, $\mathrm{d} \mathbf{L} / \mathrm{dt}=\mathbf{0}$, which is the desired result. 
2. Angular Momentum. Just as above, since $\Gamma_{\varphi}=\emptyset$, an admissible variation is

$$
\delta \varphi(\mathbf{X})=\boldsymbol{\xi} \times \boldsymbol{\varphi}
$$

again with $\boldsymbol{\xi} \in \mathbb{R}^{3}$. This results in

$$
\operatorname{GRAD}_{\mathbf{X}}[\delta \boldsymbol{\varphi}]=\hat{\boldsymbol{\xi}} \mathbf{F}
$$

where $\hat{\boldsymbol{\xi}} \in s o(3)$ is the skew-symmetric tensor with axial vector $\boldsymbol{\xi}$. More precisely, $\hat{\boldsymbol{\xi}}$ is defined by $\boldsymbol{\xi} \mathbf{a}=(\boldsymbol{\xi} \times \mathbf{a}) \forall \mathbf{a} \in \mathbb{R}^{3}$. Then, using Definition 2.3 and equation (6), one obtains

$$
\begin{aligned}
\boldsymbol{\xi} \cdot \frac{\mathrm{d}}{\mathrm{dt}} \mathbf{J} & =\boldsymbol{\xi} \cdot \frac{\mathrm{d}}{\mathrm{dt}} \int_{\Omega_{0}}(\boldsymbol{\varphi} \times \boldsymbol{\pi}) \mathrm{d} \Omega_{0} \\
& =\boldsymbol{\xi} \cdot \int_{\Omega_{0}}(\dot{\boldsymbol{\varphi}} \times \boldsymbol{\pi}+\boldsymbol{\varphi} \times \dot{\boldsymbol{\pi}}) \mathrm{d} \Omega_{0} \\
& =\boldsymbol{\xi} \cdot \int_{\Omega_{0}}(\boldsymbol{\varphi} \times \dot{\boldsymbol{\pi}}) \mathrm{d} \Omega_{0} \\
& =\int_{\Omega_{0}}(\boldsymbol{\xi} \times \boldsymbol{\varphi}) \cdot \dot{\boldsymbol{\pi}} \mathrm{d} \Omega_{0} \\
& =-\langle\mathrm{GRAD} \mathbf{X}[\boldsymbol{\xi} \times \boldsymbol{\varphi}], \mathbf{F S}\rangle_{\Omega_{0}} \\
& =-\langle\hat{\boldsymbol{\xi}} \mathbf{F}, \mathbf{F S}\rangle_{\Omega_{0}} \\
& =-\langle\hat{\boldsymbol{\xi}}, \mathbf{F S F}\rangle_{\Omega_{0}} \\
& \left.=0 \forall \boldsymbol{\xi} \in \mathbb{R}^{3} \text { (by the symmetry of } \mathbf{S}\right) .
\end{aligned}
$$

Thus, the desired result, $\mathrm{d} \mathbf{J} / \mathrm{dt}=\mathbf{0}$, follows. The conservation of angular momentum is a direct result of the invariance of the equations of motion under the variations (17), the action of the rotation group $S O(3)$.

3. Energy. Finally, consider variations of the form

$$
\delta \varphi(\mathbf{X})=\mathbf{v}
$$

which are admissible when $\Gamma_{\varphi}=\emptyset$ (or when $\bar{\varphi}=\mathbf{0}$ ). This choice shows that the time 
rate of change of the total energy is zero,

$$
\begin{aligned}
\frac{\mathrm{d}}{\mathrm{dt}} \mathrm{H} & =\frac{\mathrm{d}}{\mathrm{dt}} \mathrm{T}+\frac{\mathrm{d}}{\mathrm{dt}} \mathrm{V} \\
& =\langle\mathbf{v}, \dot{\boldsymbol{\pi}}\rangle_{\Omega_{0}}+\int_{\Omega_{0}} \dot{W}(\mathbf{C}) \mathrm{d} \Omega_{0} \\
& \left.=\langle\mathbf{v}, \dot{\boldsymbol{\pi}}\rangle_{\Omega_{0}}+\int_{\Omega_{0}} \frac{1}{2} \mathbf{S}: \dot{\mathbf{C}} \mathrm{d} \Omega_{0} \text { (by equation }(8)\right) \\
& =\langle\mathbf{v}, \dot{\boldsymbol{\pi}}\rangle_{\Omega_{0}}+\int_{\Omega_{0}} \mathbf{S}: \frac{1}{2}\left(\dot{\mathbf{F}}^{T} \mathbf{F}+\mathbf{F}^{T} \dot{\mathbf{F}}\right) \mathrm{d} \Omega_{0} \\
& =\langle\mathbf{v}, \dot{\boldsymbol{\pi}}\rangle_{\Omega_{0}}+\int_{\Omega_{0}} \mathbf{S}: \mathbf{F}^{T} \dot{\mathbf{F}} \mathrm{d} \Omega_{0} \quad(\text { symmetry of } \mathbf{S}) \\
& =\langle\mathbf{v}, \dot{\boldsymbol{\pi}}\rangle_{\Omega_{0}}+\int_{\Omega_{0}} \mathbf{S}: \mathbf{F}^{T} \mathrm{GRAD} \mathbf{X}[\mathbf{v}] \mathrm{d} \Omega_{0} \\
& =\langle\mathbf{v}, \dot{\boldsymbol{\pi}}\rangle_{\Omega_{0}}+\int_{\Omega_{0}} \mathbf{F S}: \mathrm{GRAD}_{\mathbf{X}}[\mathbf{v}] \mathrm{d} \Omega_{0} \\
& =\langle\mathbf{v}, \dot{\boldsymbol{\pi}}\rangle_{\Omega_{0}}+\left\langle\mathrm{GRAD}_{\mathbf{X}}[\mathbf{v}], \mathbf{F S}\right\rangle_{\Omega_{0}} \\
& =0(\text { by equation }(6)) .
\end{aligned}
$$

\section{Remarks 2.1.}

1. It is not strictly necessary to assume $\mathbf{b}=\mathbf{0}$ and $\overline{\mathbf{T}}=\mathbf{0}$ to derive the linear and angular momentum results. In particular, linear momentum is conserved as long as

$$
\int_{\Omega_{0}} \rho_{0} \mathbf{b} \mathrm{d} \Omega_{0}+\int_{\Gamma_{\boldsymbol{\sigma}}} \overline{\mathbf{T}} \mathrm{d} \Gamma_{0}=\mathbf{0}
$$

and angular momentum is conserved when

$$
\int_{\Omega_{0}}\left(\boldsymbol{\varphi} \times \rho_{0} \mathbf{b}\right) \mathrm{d} \Omega_{0}+\int_{\Gamma_{\boldsymbol{\sigma}}}(\boldsymbol{\varphi} \times \overline{\mathbf{T}}) \mathrm{d} \Gamma_{0}=\mathbf{0} .
$$

Additionally, total energy is conserved if $\Gamma_{\boldsymbol{\varphi}}=\emptyset$, or $\overline{\boldsymbol{\varphi}}=\mathbf{0}$, and the external forces do no work,

$$
\left\langle\mathbf{v}, \rho_{0} \mathbf{b}\right\rangle_{\Omega_{0}}+\langle\mathbf{v}, \overline{\mathbf{T}}\rangle_{\Gamma_{0}}=0
$$

However, the developments herein will continue to consider only the unforced, tractionfree, pure Neumann problem.

2. Note also that conservation of angular momentum is a direct result of the symmetry of the stress tensor $\mathbf{S}$. Any constitutive model which yields a symmetric stress tensor will thus inherit the angular momentum conservation property. However, the conservation of total energy depends critically upon the existence of a thermodynamic (in)equality such as equation (8).

The goal of this work is to construct a discrete material-point method (MPM) which also satisfies these conservation properties. 


\section{The Material Point Method}

The purpose of this section is to describe the material-point method (MPM) for the problem of equation (6). After introducing notation used to describe the discrete solution, the four main steps in the MPM algorithm are presented. Following the algorithm description, conservation properties of each step are examined.

\subsection{Basic notation}

MPM consists of a generally fixed number of material points and a background grid or mesh. Let $N_{p t}>0$ denote the number of material points of the discrete simulation, and let $N_{\text {nodes }}>0$ denote the number of nodes of the background mesh. Any quantity associated with a material point is identified by a superscript $(\cdot)^{p t}$. For example, the velocity of a material point is notated as $\mathbf{v}^{p t}$. Any quantity associated with the background mesh is identified by a superscript $(\cdot)^{h}$, with $h>0$ denoting the mesh size, or by indexes $\{A, B, C \ldots\}$ indicating nodal values. For example, $\mathbf{v}^{h}(\mathbf{x})$ is the velocity field of the mesh with nodal values $\mathbf{v}_{A}$ for $A \in\left\{1,2, \ldots, N_{\text {nodes }}\right\}$. (In a slight abuse of notation, $(\cdot)^{h}$ is also used to notate the vector of nodal values of $(\cdot)$. This should not cause the reader any confusion in the context where it is used.) A subscript $(\cdot)_{n+1}$ indicates the time step index, corresponding to a solution at time $t_{n+1}$. A capital $\Delta$ is used to indicate a time step increment in a quantity, $\Delta(\cdot)=(\cdot)_{n+1}-(\cdot)_{n}$, and in standard fashion, $(\cdot)_{n+\alpha}:=(1-\alpha)(\cdot)_{n}+\alpha(\cdot)_{n+1} \forall \alpha \in[0,1]$.

Denote by $N^{A}$ the shape function of node $A$ of the mesh. Let $\mathcal{W}^{h} \subset \mathcal{W}$ denote a typical finite dimensional finite element subspace of $\mathcal{W}$, with corresponding solution space $\mathcal{S}^{h} \subset \mathcal{S}$. Then, for example, any $\delta \varphi^{h} \in \mathcal{W}^{h}$ takes the form

$$
\delta \boldsymbol{\varphi}^{h}(\mathbf{x})=\sum_{A=1}^{N_{\text {nodes }}} N^{A}(\mathbf{x}) \delta \boldsymbol{\varphi}_{A} .
$$

All integrals are evaluated by treating the material points as quadrature points. For example, the second term in (6) can be approximated as

$$
\left.\begin{array}{rl}
\mathbf{B}^{h}(\mathbf{x}):= & \operatorname{GRAD}_{\mathbf{X}}\left[\delta \varphi^{h}(\mathbf{x})\right]=\sum_{A=1}^{N_{\text {nodes }}} \delta \boldsymbol{\varphi}_{A} \otimes\left(\mathbf{F}_{n}^{T}(\mathbf{x}) \operatorname{grad}_{n}\left[N^{A}(\mathbf{x})\right]\right) \\
& \left\langle\operatorname{GRAD}_{\mathbf{X}}[\delta \boldsymbol{\varphi}], \mathbf{F}_{n+\alpha} \mathbf{S}\right\rangle_{\Omega_{0}} \approx \sum_{p t=1}^{N_{p t}}\left(\mathbf{B}^{h}\left(\mathbf{x}_{n}^{p t}\right): \mathbf{F}_{n+\alpha}^{p t} \mathbf{S}^{p t}\right) \Omega_{0}^{p t}
\end{array}\right\}
$$

where $\mathbf{x}_{n}^{p t}=\varphi_{n}^{p t}$ is the material-point position at time step $n$ and $\Omega_{0}^{p t}>0$ is the material volume associated with the material point $p t$. The transformation $\operatorname{GRAD}_{\mathbf{X}}[\cdot]=\mathbf{F}_{n}^{T} \operatorname{grad}_{n}[\cdot]$ is also used, where $\operatorname{grad}_{n}$ indicates the gradient with respect to the configuration at time $\mathrm{t}_{n}$. Figure 1 schematically shows the notation used to relate the reference configuration of the deforming body with its configuration at times $t_{n}$ and $t_{n+1}$.

The mass $m^{p t}=\rho_{0}\left(\mathbf{x}^{p t}\right) \Omega_{0}^{p t}>0$ is constant for each material point. The material-point momentum is then $\boldsymbol{\pi}^{p t}:=m^{p t} \mathbf{v}^{p t}$. Using the material-point masses, the consistent mass 


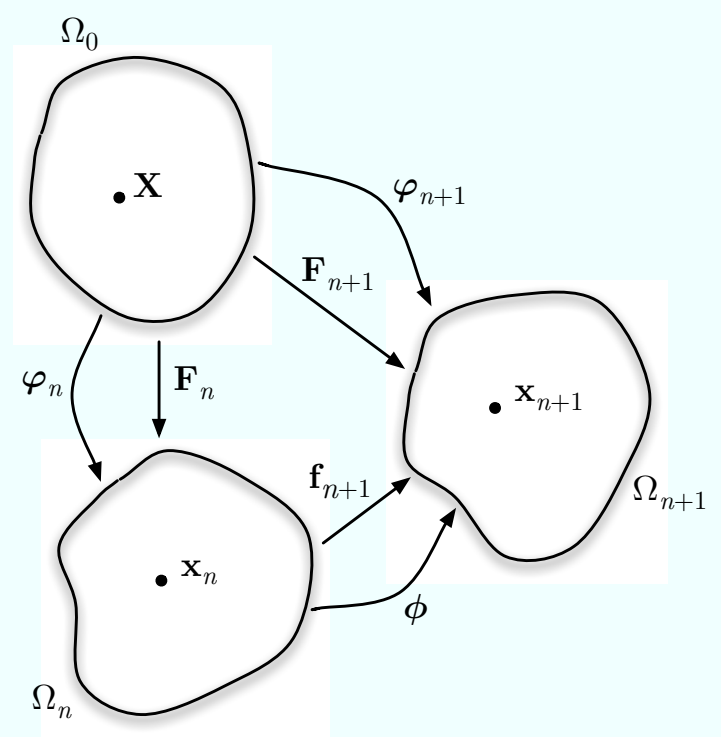

Figure 1: Diagram indicating the notation used to relate the reference configuration of the body with its configuration at times $t_{n}$ and $t_{n+1}$. By this construction, $\boldsymbol{\varphi}_{n+1}=\boldsymbol{\phi} \circ \boldsymbol{\varphi}_{n}$ and $\mathbf{F}_{n+1}=\mathbf{f}_{n+1} \mathbf{F}_{n}$. Assuming the same fixed Cartesian coordinate system for all three configurations, $\phi=\mathrm{id}+\Delta \varphi$, where $\Delta \varphi=\left(\boldsymbol{\varphi}_{n+1}-\boldsymbol{\varphi}_{n}\right) \circ \boldsymbol{\varphi}_{n}^{-1}$.

matrix $\mathbf{M}$ is defined with nodal components

$$
M^{A B}:=\sum_{p t=1}^{N_{p t}} m^{p t} N^{A}\left(\mathbf{x}^{p t}\right) N^{B}\left(\mathbf{x}^{p t}\right) \approx \int_{\Omega_{0}} \rho_{0} N^{A} N^{B} \mathrm{~d} \Omega_{0} .
$$

Analogously, the diagonal lumped mass matrix $\overline{\mathbf{M}}$ is defined with nodal components

$$
\left.\begin{array}{rl}
\bar{M}^{A B} & =\delta^{A B}\left[\sum_{p t=1}^{N_{p t}} m^{p t} N^{A}\left(\mathbf{x}^{p t}\right)\right] \\
& =\delta^{A B}\left[\sum_{B=1}^{N_{\text {nodes }}} M^{A B}\right] \approx \delta^{A B}\left[\int_{\Omega_{0}} \rho_{0} N^{A} \mathrm{~d} \Omega_{0}\right]
\end{array}\right\} \quad(\text { no sum on } A) .
$$

An effective mass matrix $\widetilde{\mathbf{M}}$ is defined as the convex combination of the consistent and lumped masses,

$$
\widetilde{\mathbf{M}}:=(1-\varepsilon) \mathbf{M}+\varepsilon \overline{\mathbf{M}} \quad \text { for } \varepsilon \in[0,1] .
$$

\section{Remarks 3.1.}

1. In practice, the integrals and sums above are evaluated on an element by element basis. Since every element contains a finite number of material points, the integration and assembly process is a standard finite element procedure [26], [47].

2. If the mass density is viewed as a sum of point masses, $\rho_{0}=\sum_{p t=1}^{N_{p t}} m^{p t} \delta\left(\mathbf{X}-\mathbf{X}^{p t}\right)$, then the evaluation of the integral in (24) is exact. 
3. The presence of the subscript $n$ on the gradient in equation $(23)_{1}$ is significant. The material-point method is implemented as an updated Lagrangian finite element method [8], [9] and as such the grid (mesh) $\Omega_{n}$ is always used as an updated reference configuration for all gradient evaluations. The incremental motion (see Figure 1) is defined by a $\operatorname{map} \phi: \Omega_{n} \rightarrow \Omega_{n+1}$ with $\boldsymbol{\phi}=\mathbf{i d}+\Delta \boldsymbol{\varphi}$, such that $\mathbf{f}_{n+1}:=\operatorname{grad}_{n}[\boldsymbol{\phi}]$ and $\mathbf{F}_{n+1}=\mathbf{f}_{n+1} \mathbf{F}_{n}$. In this regard the incremental kinematics used in MPM are exactly the same as those used the in incremental flow formulation ([9], Sections 2.2 and 3.1).

4. It is necessary to consider a general effective mass matrix since there exist materialpoint configurations, relative to a given grid, for which the consistent mass matrix is singular. This point is discussed in Appendix A of [16]. As in standard finite element methods, reduced integration of the mass matrix (eg. one-point quadrature) can result in a singular matrix. The possible singularity of the consistent mass matrix is a direct result of the non-optimal quadrature rule generated by using the material points as quadrature points. However, the lumped mass matrix is positive-definite and the consistent mass matrix is positive semi-definite. Thus the effective mass matrix is always positive-definite for $\varepsilon>0$.

\subsection{Time stepping algorithm}

Given the material-point data $\left\{\boldsymbol{\varphi}_{n}^{p t}, \mathbf{v}_{n}^{p t}, \mathbf{F}_{n}^{p t}\right\}$ at time $t_{n}$, the goal is to determine the materialpoint data $\left\{\boldsymbol{\varphi}_{n+1}^{p t}, \mathbf{v}_{n+1}^{p t}, \mathbf{F}_{n+1}^{p t}\right\}$ at time $\mathrm{t}_{n+1}:=\mathrm{t}_{n}+\Delta \mathrm{t}$. Assuming a background finite element mesh exists, the MPM algorithm is as follows:

Algorithm MPM. The algorithm consists of four primary steps:

1. Material-Point-to-Grid Projection. Compute the effective mass matrix $\widetilde{\mathbf{M}}_{n}$ using equations (24), (25) and (26). For example, the consistent mass matrix for this time step is

$$
M_{n}^{A B}=\sum_{p t=1}^{N_{p t}} m^{p t} N^{A}\left(\mathbf{x}_{n}^{p t}\right) N^{B}\left(\mathbf{x}_{n}^{p t}\right) .
$$

Compute the grid velocities $\mathbf{v}_{n}^{h}$ such that

$$
\sum_{B=1}^{N_{\text {nodes }}} \widetilde{M}_{n}^{A B} \mathbf{v}_{B, n}=\sum_{p t=1}^{N_{p t}} N^{A}\left(\mathbf{x}_{n}^{p t}\right) \boldsymbol{\pi}_{n}^{p t} \forall A \in\left\{1,2, \ldots, N_{\text {nodes }}\right\} .
$$

2. Lagrangian Mesh Dynamics. (Reference [40], Section 4.1.1. See also Section 44 in [39].) Solve the nonlinear algebraic equations

$$
\left.\begin{array}{r}
\frac{1}{\Delta \mathrm{t}} \sum_{B=1}^{N_{\text {nodes }}} \widetilde{M}_{n}^{A B}\left(\mathbf{v}_{B, n+1}-\mathbf{v}_{B, n}\right)+\left(\mathbf{F}^{i n t}\right)_{n+1 / 2}^{A}-\left(\mathbf{F}^{e x t}\right)_{n+1 / 2}^{A}=\mathbf{0} \\
\frac{1}{\Delta \mathrm{t}} \Delta \boldsymbol{\varphi}_{A}=\mathbf{v}_{A, n+1 / 2}
\end{array}\right\}
$$


for $A \in\left\{1,2, \ldots, N_{\text {nodes }}\right\}$, where $\left(\mathbf{F}^{i n t}\right)_{n+1 / 2}^{A}$ and $\left(\mathbf{F}^{\text {ext }}\right)_{n+1 / 2}^{A}$ are the internal and external forces, respectively, associated with node $A$ at time $t_{n+1 / 2}$. These forces are defined as

$$
\left.\begin{array}{rl}
\left(\mathbf{F}^{i n t}\right)_{n+1 / 2}^{A} & :=\sum_{p t=1}^{N_{p t}}\left(\mathbf{F}_{n+1 / 2}^{p t} \mathbf{S}^{p t}\right)\left(\mathbf{F}_{n}^{p t}\right)^{T} \operatorname{grad}_{n}\left[N^{A}\left(\mathbf{x}_{n}^{p t}\right)\right] \Omega_{0}^{p t} \\
& \approx \int_{\Omega_{0}}\left(\mathbf{F}_{n+1 / 2} \mathbf{S}\right) \operatorname{GRAD}_{\mathbf{X}}\left[N^{A}\right] \mathrm{d} \Omega_{0}
\end{array}\right\},
$$

where $\overline{\mathbf{T}}_{n+1 / 2}^{A}$ is an approximation to the nodal value of the surface traction, $\overline{\mathbf{T}}_{n+1 / 2}^{A} \approx$ $\int_{\Gamma_{\sigma}} N^{A} \overline{\mathbf{T}}_{n+1 / 2} \mathrm{~d} \Gamma_{0}$. The sums in equation (30) arise as in equation (23), as a sum of material-point (integration point) contributions approximating the integral over each finite element. During this step, the deformation gradient for each material point is multiplicatively updated via

$$
\left.\begin{array}{r}
\mathbf{f}_{n+1}^{h}\left(\mathbf{x}_{n}\right):=\mathbf{I}+\operatorname{grad}_{n}\left[\Delta \varphi^{h}\left(\mathbf{x}_{n}\right)\right] \\
\mathbf{F}_{n+1}^{p t}:=\mathbf{f}_{n+1}^{h}\left(\mathbf{x}_{n}^{p t}\right) \mathbf{F}_{n}^{p t}
\end{array}\right\}
$$

The algorithmic stress $\mathbf{S}^{p t}$ remains as yet undefined, but is assumed symmetric. This tensor shall be defined so that energy is exactly conserved. The above equations of this step, all together, are the Galerkin spatial projection of the weak form (6) onto the finite element subspace, coupled with an incremental midpoint-based time stepping scheme.

3. Material-Point Update. Update the material-point positions and velocities via

$$
\left.\begin{array}{l}
\boldsymbol{\varphi}_{n+1}^{p t}=\boldsymbol{\varphi}_{n}^{p t}+\Delta \boldsymbol{\varphi}^{h}\left(\mathbf{x}_{n}^{p t}\right) \\
\mathbf{v}_{n+1}^{p t}= \\
\mathbf{v}_{n}^{p t}+\Delta \mathbf{v}^{h}\left(\mathbf{x}_{n}^{p t}\right)
\end{array}\right\}
$$

4. Regrid. During step 2, the finite element mesh deforms. During step 4, the mesh may be moved, if desired. In particular, the mesh may be returned to the position it occupied at the beginning of step 2. This choice is typical of many MPM implementations. Alternatively, the mesh may be redefined to suit any requirements the user may have, including the enforcement of Dirichlet boundary conditions, or adaption to features of the solution. 


\section{Remarks 3.2.}

1. Imposed displacements and traction boundary conditions are applied to the finite element mesh during step 2 above. Imposed displacements and boundary forces are not applied to the material points directly. Of course, application of boundary conditions is a standard finite element procedure which involves only the nodes, and not the integration points [26], [47].

2. The material-point-to-grid projection in step 1 may also be affected by boundary conditions. Consider, for example, the situation where the displacement (and consequently the velocity) is prescribed on some part of the mesh boundary. Those boundary velocities should not be determined by projection, but rather directly by discrete time differentiation. This creates a constraint on the projection (28).

3. During the Lagrangian step 2, the mass matrix is constant.

4. The incremental displacement field $\Delta \varphi^{h}$ is typically used as the primary solution variable (and Newton iterate) during step 2. At the beginning of the step it is initialized to zero (0). Thus the initial condition for the velocity field becomes

$$
\underbrace{\Delta \boldsymbol{\varphi}^{h}=\mathbf{0} \Longleftrightarrow \mathbf{v}_{n+1 / 2}^{h}=\mathbf{0} \Longleftrightarrow \mathbf{v}_{n+1}^{h}=-\mathbf{v}_{n}^{h}}_{\text {initial conditions }} .
$$

Clearly the choice of initial conditions for the mesh dynamics is not unique. Said choice should, however, be consistent with the time integration $(29)_{2}$.

5. During step 3, only changes in the displacement and velocity are mapped to the material points. Mapping only changes in quantities is introduced in [13] in order to keep dissipation small when a lumped mass matrix is used.

6. Generally, step 4 above involves a search. Every material point must be located within some finite element and the corresponding natural coordinates must be computed. For arbitrary distorted elements this typically involves inverting the isoparametric mapping using a Newton algorithm [31].

7. Since the material points serve as quadrature points for the finite elements of the background mesh, MPM can be viewed as an extension of the finite element method. If step 4 is omitted, and the initial material-point positions and volumes correspond to optimally located gauss quadrature points for each element, the MPM algorithm essentially collapses to a standard (updated) Lagrangian finite element analysis.

\subsection{Conservation properties}

After defining the discrete analogs of the the continuum conserved quantities, each step of MPM is examined to assess its conservation properties. 
Definition 3.1. The total grid (mesh) linear momentum $\mathbf{L}^{h}$ is defined as

$$
\mathbf{L}^{h}:=\sum_{A=1}^{N_{\text {nodes }}}\left(\sum_{B=1}^{N_{\text {nodes }}} \widetilde{M}^{A B} \mathbf{v}_{B}\right)=\sum_{A=1}^{N_{\text {nodes }}} \bar{M}^{A A} \mathbf{v}_{A} .
$$

Definition 3.2. The total material-point linear momentum $\mathbf{L}^{p t}$ is defined as

$$
\mathbf{L}^{p t}:=\sum_{p t=1}^{N_{p t}} \boldsymbol{\pi}^{p t}
$$

Definition 3.3. The total grid (mesh) angular momentum $\mathbf{J}^{h}$ is defined as

$$
\mathbf{J}^{h}:=\sum_{A=1}^{N_{\text {nodes }}}\left(\boldsymbol{\varphi}_{A} \times \sum_{B=1}^{N_{\text {nodes }}} \widetilde{M}^{A B} \mathbf{v}_{B}\right) .
$$

Definition 3.4. The total material-point angular momentum $\mathbf{J}^{\text {pt }}$ is defined as

$$
\mathbf{J}^{p t}:=\sum_{p t=1}^{N_{p t}}\left(\boldsymbol{\varphi}^{p t} \times \boldsymbol{\pi}^{p t}\right)=\sum_{p t=1}^{N_{p t}}\left(\mathbf{x}^{p t} \times \boldsymbol{\pi}^{p t}\right) .
$$

Definition 3.5. The total grid (mesh) kinetic energy $\mathrm{T}^{h}$ is defined as

$$
2 \mathrm{~T}^{h}:=\sum_{A=1}^{N_{\text {nodes }}}\left(\mathbf{v}_{A} \cdot \sum_{B=1}^{N_{\text {nodes }}} \widetilde{M}^{A B} \mathbf{v}_{B}\right)
$$

Definition 3.6. The total material-point kinetic energy $\mathrm{T}^{p t}$ is defined as

$$
2 \boldsymbol{\top}^{p t}:=\sum_{p t=1}^{N_{p t}}\left(\boldsymbol{\pi}^{p t} \cdot \frac{1}{m^{p t}} \boldsymbol{\pi}^{p t}\right)=\sum_{p t=1}^{N_{p t}}\left(\boldsymbol{\pi}^{p t} \cdot \mathbf{v}^{p t}\right)=\sum_{p t=1}^{N_{p t}} m^{p t}\left\|\mathbf{v}^{p t}\right\|^{2}
$$

Definition 3.7. The total material-point potential energy $\mathrm{V}^{p t}$ is defined as

$$
\bigvee^{p t}:=\sum_{p t=1}^{N_{p t}} W\left(\mathbf{C}^{p t}\right) \Omega_{0}^{p t}
$$

Definition 3.8. The total grid (mesh) energy $\mathrm{H}^{h}$ is defined as

$$
\mathrm{H}^{h}:=\mathrm{T}^{h}+\mathrm{V}^{p t}
$$


Definition 3.9. The total material-point energy $\mathrm{H}^{p t}$ is defined as

$$
\mathrm{H}^{p t}:=\mathrm{T}^{p t}+\mathrm{V}^{p t}
$$

There is no need to draw a distinction between total potential energy defined on the grid and total potential energy defined on the material points. They are in fact the same quantity. To compute the total grid potential energy one would use the material points as quadrature points for the integral, and the result would be exactly $\mathrm{V}^{p t}$.

Before proceeding with the conservation properties of MPM, the following lemma is established for later use.

Lemma 3.1. The symmetric matrix $(\overline{\mathbf{M}}-\mathbf{M})$ is positive-semidefinite.

Proof. Any eigenvalue, $\lambda$, of the matrix in question, lies in the union of its Gershgorin disks [27]. The $A^{\text {th }}$ disk is $z \in \mathbb{C}$, such that $\left|z-\left(\bar{M}^{A A}-M^{A A}\right)\right| \leq \bar{M}^{A A}-M^{A A}$ (no sum on $A)$. These disks all lie in the right-half plane, $\operatorname{Re}(z) \geq 0$. Since $\lambda$ is real, it must be that $\lambda \geq 0$.

\subsubsection{The material-point-to-grid projection}

The projection of data from the material points to the grid preserves the total linear and angular momentum, but the total kinetic energy on the grid is less than the total kinetic energy in the material-point representation of the solution. These properties are noted in [12] where the lumped mass matrix is used, and in [16] where the consistent mass matrix is used. Angular momentum is also discussed in [11] using the lumped mass matrix. Proofs and discussion of these points follow.

Proposition 3.2. The material-point-to-grid velocity projection conserves total linear momentum, $\mathbf{L}_{n}^{h}=\mathbf{L}_{n}^{p t}$.

Proof.

$$
\begin{aligned}
\mathbf{L}_{n}^{h} & =\sum_{A=1}^{N_{\text {nodes }}}\left(\sum_{B=1}^{N_{\text {nodes }}} \widetilde{M}_{n}^{A B} \mathbf{v}_{B, n}\right) \\
& =\sum_{A=1}^{N_{\text {nodes }}}\left(\sum_{p t=1}^{N_{p t}} N^{A}\left(\mathbf{x}_{n}^{p t}\right) \boldsymbol{\pi}_{n}^{p t}\right) \quad(\text { by }(28)) \\
& =\sum_{p t=1}^{N_{p t}}\left(\sum_{A=1}^{N_{\text {nodes }}} N^{A}\left(\mathbf{x}_{n}^{p t}\right)\right) \boldsymbol{\pi}_{n}^{p t} \\
& =\sum_{p t=1}^{N_{p t}} \boldsymbol{\pi}_{n}^{p t} \\
& =\mathbf{L}_{n}^{p t} .
\end{aligned}
$$


Proposition 3.3. The material-point-to-grid velocity projection conserves total angular momentum, $\mathbf{J}_{n}^{h}=\mathbf{J}_{n}^{p t}$.

Proof.

$$
\begin{aligned}
\mathbf{J}_{n}^{h} & =\sum_{A=1}^{N_{\text {nodes }}}\left(\boldsymbol{\varphi}_{A, n} \times \sum_{B=1}^{N_{\text {nodes }}} \widetilde{M}_{n}^{A B} \mathbf{v}_{B, n}\right) \\
& =\sum_{A=1}^{N_{\text {nodes }}}\left(\boldsymbol{\varphi}_{A, n} \times \sum_{p t=1}^{N_{p t}} N^{A}\left(\mathbf{x}_{n}^{p t}\right) \boldsymbol{\pi}_{n}^{p t}\right) \\
& =\sum_{p t=1}^{N_{p t}}\left(\sum_{A=1}^{N_{\text {nodes }}} N^{A}\left(\mathbf{x}_{n}^{p t}\right) \boldsymbol{\varphi}_{A, n}\right) \times \boldsymbol{\pi}_{n}^{p t} \\
& =\sum_{p t=1}^{N_{p t}} \boldsymbol{\varphi}_{n}^{p t} \times \boldsymbol{\pi}_{n}^{p t} \\
& =\mathbf{J}_{n}^{p t} .
\end{aligned}
$$

Proposition 3.4. The material-point-to-grid velocity projection dissipates kinetic energy, $\mathrm{T}_{n}^{p t} \geq \mathrm{T}_{n}^{h}$.

Proof. Define

$$
\mathcal{E}_{v}:=\sum_{p t=1}^{N_{p t}} m^{p t}\left\|\mathbf{v}_{n}^{p t}-\mathbf{v}_{n}^{h}\left(\mathbf{x}_{n}^{p t}\right)\right\|^{2} \geq 0,
$$

as a measure of velocity projection error. Next, one may write

$$
\mathbf{v}_{n}^{p t}=\mathbf{v}_{n}^{p t}-\mathbf{v}_{n}^{h}\left(\mathbf{x}_{n}^{p t}\right)+\mathbf{v}_{n}^{h}\left(\mathbf{x}_{n}^{p t}\right),
$$

so that

$$
\begin{aligned}
2 \mathbf{T}_{n}^{p t} & =\sum_{p t=1}^{N_{p t}} m^{p t}\left\|\mathbf{v}_{n}^{p t}\right\|^{2} \\
& =\sum_{p t=1}^{N_{p t}} m^{p t}\left\|\left(\mathbf{v}_{n}^{p t}-\mathbf{v}_{n}^{h}\left(\mathbf{x}_{n}^{p t}\right)\right)+\mathbf{v}_{n}^{h}\left(\mathbf{x}_{n}^{p t}\right)\right\|^{2} \\
& =\mathcal{E}_{v}+\sum_{p t=1}^{N_{p t}} m^{p t}\left[2\left(\mathbf{v}_{n}^{p t}-\mathbf{v}_{n}^{h}\left(\mathbf{x}_{n}^{p t}\right)\right) \cdot \mathbf{v}_{n}^{h}\left(\mathbf{x}_{n}^{p t}\right)+\mathbf{v}_{n}^{h}\left(\mathbf{x}_{n}^{p t}\right) \cdot \mathbf{v}_{n}^{h}\left(\mathbf{x}_{n}^{p t}\right)\right] \\
& =\mathcal{E}_{v}+2 \sum_{p t=1}^{N_{p t}} m^{p t} \mathbf{v}_{n}^{p t} \cdot \mathbf{v}_{n}^{h}\left(\mathbf{x}_{n}^{p t}\right)-\sum_{p t=1}^{N_{p t}} m^{p t} \mathbf{v}_{n}^{h}\left(\mathbf{x}_{n}^{p t}\right) \cdot \mathbf{v}_{n}^{h}\left(\mathbf{x}_{n}^{p t}\right)
\end{aligned}
$$


The second term on the right-hand side of (46) can be evaluated as

$$
\begin{aligned}
2 \sum_{p t=1}^{N_{p t}} m^{p t} \mathbf{v}_{n}^{p t} \cdot \mathbf{v}_{n}^{h}\left(\mathbf{x}_{n}^{p t}\right) & =2 \sum_{p t=1}^{N_{p t}}\left(m^{p t} \mathbf{v}_{n}^{p t} \cdot\left(\sum_{A=1}^{N_{\text {nodes }}} N^{A}\left(\mathbf{x}_{n}^{p t}\right) \mathbf{v}_{A, n}\right)\right) \\
& =2 \sum_{A=1}^{N_{\text {nodes }}}\left(\mathbf{v}_{A, n} \cdot\left(\sum_{p t=1}^{N_{p t}} m^{p t} \mathbf{v}_{n}^{p t} N^{A}\left(\mathbf{x}_{n}^{p t}\right)\right)\right) \\
& =2 \sum_{A=1}^{N_{\text {nodes }}}\left(\mathbf{v}_{A, n} \cdot \sum_{B=1}^{N_{\text {nodes }}} \widetilde{M}_{n}^{A B} \mathbf{v}_{B, n}\right) \quad(\text { by } \\
& =4 \mathrm{~T}_{n}^{h} .
\end{aligned}
$$

The third term on the right-hand side of (46) can be evaluated as

$$
\begin{aligned}
\sum_{p t=1}^{N_{p t}} m^{p t} \mathbf{v}_{n}^{h}\left(\mathbf{x}_{n}^{p t}\right) \cdot \mathbf{v}_{n}^{h}\left(\mathbf{x}_{n}^{p t}\right) & =\sum_{p t=1}^{N_{p t}} m^{p t}\left(\sum_{A=1}^{N_{\text {nodes }}} N^{A}\left(\mathbf{x}_{n}^{p t}\right) \mathbf{v}_{A, n}\right) \cdot\left(\sum_{B=1}^{N_{\text {nodes }}} N^{B}\left(\mathbf{x}_{n}^{p t}\right) \mathbf{v}_{B, n}\right) \\
& =\sum_{A=1}^{N_{\text {nodes }}} \sum_{B=1}^{N_{\text {nodes }}} \mathbf{v}_{A, n} \cdot \mathbf{v}_{B, n}\left(\sum_{p t=1}^{N_{p t}} m^{p t} N^{A}\left(\mathbf{x}_{n}^{p t}\right) N^{B}\left(\mathbf{x}_{n}^{p t}\right)\right) \\
& =\sum_{A=1}^{N_{\text {nodes }}} \sum_{B=1}^{N_{\text {nodes }}} \mathbf{v}_{A, n} \cdot M_{n}^{A B} \mathbf{v}_{B, n} \\
& =\mathbf{v}_{n}^{h} \cdot \mathbf{M} \mathbf{v}_{n}^{h},
\end{aligned}
$$

which is twice the consistent mass, grid kinetic energy. Thus equation (46) reduces to

$$
\begin{aligned}
2 \mathbf{T}_{n}^{p t} & =\mathcal{E}_{v}+4 \mathrm{~T}_{n}^{h}-\left(\mathbf{v}_{n}^{h} \cdot \mathbf{M} \mathbf{v}_{n}^{h}\right) \\
& =2 \mathbf{T}_{n}^{h}+\left[\left(\mathbf{v}_{n}^{h} \cdot \widetilde{\mathbf{M}} \mathbf{v}_{n}^{h}\right)-\left(\mathbf{v}_{n}^{h} \cdot \mathbf{M} \mathbf{v}_{n}^{h}\right)\right]+\mathcal{E}_{v} \quad \text { (by Definition 3.5) } \\
& =2 \mathrm{~T}_{n}^{h}+\left[\mathbf{v}_{n}^{h} \cdot(\widetilde{\mathbf{M}}-\mathbf{M}) \mathbf{v}_{n}^{h}\right]+\mathcal{E}_{v} \\
& =2 \mathrm{~T}_{n}^{h}+\underbrace{\varepsilon\left[\mathbf{v}_{n}^{h} \cdot(\overline{\mathbf{M}}-\mathbf{M}) \mathbf{v}_{n}^{h}\right]}_{\geq 0}+\underbrace{\mathcal{E}_{v}}_{\geq 0}(\text { by }(26))
\end{aligned}
$$

Now, by Lemma 3.1, the second term on the right-hand side above is positive, and by construction $\mathcal{E}_{v}$ is positive. This produces the conclusion that $\mathrm{T}_{n}^{p t} \geq \mathrm{T}_{n}^{h}$, which is the desired result.

\section{Remarks 3.3.}

1. Total linear and angular momentum are transferred without error from the material points to the grid for any choice of the effective mass matrix.

2. For kinetic energy, if the consistent mass matrix is used, then $\varepsilon=0$, and the second term on the right-hand side of equation (49) vanishes. Otherwise this term is $\mathcal{O}(h)$. If the grid is Lagrangian, that is, if step 4 of the MPM algorithm is not done, then 
$\mathcal{E}_{v}=0$. Thus, nonzero $\mathcal{E}_{v}$ arises from the motion of the grid relative to the material points, and is the typical case in the MPM. When $\mathcal{E}_{v} \neq 0$ or $\varepsilon \neq 0$, not all kinetic energy is transferred from the material points to the grid at the start of a new time step.

3. Since not all energy is transferred from the material points to the grid, the grid representation of the solution has less energy than the material point description of the solution. Typical remeshing algorithms used with standard finite element analyses dissipate kinetic energy when the solution on one mesh is replaced with the solution on a different mesh. In MPM total energy is not affected by the discrepancy (49) between the grid energy and the material-point energy because the material-point description is retained throughout the computation. Thus the energy in the material-point representation of the solution is not lost. Of course, accuracy of the energy transfer is important for the overall performance of the algorithm.

\subsubsection{The Lagrangian mesh dynamics}

Once information from the material points has been projected onto a mesh, standard finite element techniques can be used to solve the momentum equation on that mesh. Momentum and energy conserving finite element algorithms have been derived previously in the references [2], [3], [21] and [40]. Linear and angular momentum conservation are fairly straightforward and the fact that these quantities are conserved is stated in the next two propositions. For completeness, the proofs of these propositions are provided in the Appendix. Conservation of energy is less straightforward and is discussed below. As shown in the references, the key to constructing such a method is the proper choice of the algorithmic stress $\mathbf{S}^{p t}$.

Proposition 3.5. Assume $\left(\mathbf{F}^{e x t}\right)_{n+1 / 2}^{A}=\mathbf{0}$. Then the mesh dynamics conserve exactly the total mesh linear momentum, $\Delta \mathbf{L}^{h}=0$.

Proof. See Appendix A.

Proposition 3.6. Assume $\left(\mathbf{F}^{e x t}\right)_{n+1 / 2}^{A}=\mathbf{0}$. Then the mesh dynamics conserve exactly the total mesh angular momentum, $\Delta \mathbf{J}^{h}=0$.

Proof. See Appendix A.

Remarks 3.4. As in the continuum case, the conservation of angular momentum depends only upon the symmetry of the tensor $\mathbf{S}^{p t}$. This tensor must still be defined to ensure discrete energy conservation.

Proposition 3.7. Assume $\left(\mathbf{F}^{e x t}\right)_{n+1 / 2}^{A}=\mathbf{0}$. Additionally, assume that the algorithmic stress satisfies the discrete dissipation (in)equality

$$
\frac{1}{2} \mathbf{S}^{p t}: \Delta \mathbf{C}^{p t}=\frac{1}{2} \mathbf{S}^{p t}:\left(\mathbf{C}_{n+1}^{p t}-\mathbf{C}_{n}^{p t}\right)=W\left(\mathbf{C}_{n+1}^{p t}\right)-W\left(\mathbf{C}_{n}^{p t}\right) .
$$

Then the mesh dynamics exactly conserves the total mesh energy, $\mathrm{H}_{n+1}^{h}=\mathrm{H}_{n}^{h}$, so that $\Delta \mathrm{H}^{h}=\Delta \mathrm{T}^{h}+\Delta \mathrm{V}^{h}=0$. 
Proof. The presentation follows closely that of Proposition 4.1 in [3] and Proposition 2 in [21], except that the effective mass matrix is used. First, since $\widetilde{\mathbf{M}}$ is symmetric, note that the change in kinetic energy can be written

$$
\begin{aligned}
\Delta \mathrm{T}^{h} & =\mathrm{T}_{n+1}^{h}-\mathrm{T}_{n}^{h} \\
& =\frac{1}{2} \sum_{A, B=1}^{N_{\text {nodes }}}\left(\mathbf{v}_{A, n}+\mathbf{v}_{A, n+1}\right) \cdot \widetilde{M}_{n}^{A B}\left(\mathbf{v}_{B, n+1}-\mathbf{v}_{B, n}\right) \\
& =\sum_{A, B=1}^{N_{\text {nodes }}} \mathbf{v}_{A, n+1 / 2} \cdot \widetilde{M}_{n}^{A B}\left(\mathbf{v}_{B, n+1}-\mathbf{v}_{B, n}\right) .
\end{aligned}
$$


Continuing, the change in potential energy is

$$
\begin{aligned}
& \Delta \mathrm{V}^{p t}=\mathrm{V}_{n+1}^{p t}-\mathrm{V}_{n}^{p t} \\
& =\sum_{p t=1}^{N_{p t}}\left[W\left(\mathbf{C}_{n+1}^{p t}\right)-W\left(\mathbf{C}_{n}^{p t}\right)\right] \Omega_{0}^{p t} \\
& =\sum_{p t=1}^{N_{p t}} \frac{1}{2}\left(\mathbf{C}_{n+1}^{p t}-\mathbf{C}_{n}^{p t}\right): \mathbf{S}^{p t} \Omega_{0}^{p t} \\
& =\sum_{p t=1}^{N_{p t}} \frac{1}{2}[\mathbf{C}_{n+1}^{p t}-\mathbf{C}_{n}^{p t}+\underbrace{\left(\mathbf{F}_{n}^{p t}\right)^{T} \mathbf{F}_{n+1}^{p t}-\left(\mathbf{F}_{n+1}^{p t}\right)^{T} \mathbf{F}_{n}^{p t}}_{\text {skew }}]: \mathbf{S}^{p t} \Omega_{0}^{p t} \\
& =\sum_{p t=1}^{N_{p t}} \frac{1}{2}\left(\mathbf{F}_{n+1}^{p t}+\mathbf{F}_{n}^{p t}\right)^{T}\left(\mathbf{F}_{n+1}^{p t}-\mathbf{F}_{n}^{p t}\right): \mathbf{S}^{p t} \Omega_{0}^{p t} \\
& =\sum_{p t=1}^{N_{p t}}\left(\mathbf{F}_{n+1 / 2}^{p t}\right)^{T}\left(\mathbf{F}_{n+1}^{p t}-\mathbf{F}_{n}^{p t}\right): \mathbf{S}^{p t} \Omega_{0}^{p t} \\
& =\sum_{p t=1}^{N_{p t}}\left(\mathbf{F}_{n+1}^{p t}-\mathbf{F}_{n}^{p t}\right):\left(\mathbf{F}_{n+1 / 2}^{p t} \mathbf{S}^{p t}\right) \Omega_{0}^{p t} \\
& =\sum_{p t=1}^{N_{p t}}\left(\operatorname{grad}_{n}\left[\Delta \varphi^{h}\left(\mathbf{x}_{n}^{p t}\right)\right] \mathbf{F}_{n}^{p t}\right):\left(\mathbf{F}_{n+1 / 2}^{p t} \mathbf{S}^{p t}\right) \Omega_{0}^{p t} \\
& =\sum_{p t=1}^{N_{p t}} \sum_{A=1}^{N_{\text {nodes }}}\left(\left[\Delta \boldsymbol{\varphi}_{A} \otimes \operatorname{grad}_{n}\left[N^{A}\left(\mathbf{x}_{n}^{p t}\right)\right]\right] \mathbf{F}_{n}^{p t}\right):\left(\mathbf{F}_{n+1 / 2}^{p t} \mathbf{S}^{p t}\right) \Omega_{0}^{p t} \\
& =\sum_{A=1}^{N_{\text {nodes }}} \sum_{p t=1}^{N_{p t}} \Delta \boldsymbol{\varphi}_{A} \cdot\left(\mathbf{F}_{n+1 / 2}^{p t} \mathbf{S}^{p t}\right) \underbrace{\left(\mathbf{F}_{n}^{p t}\right)^{T} \operatorname{grad}_{n}\left[N^{A}\left(\mathbf{x}_{n}^{p t}\right)\right]}_{\operatorname{GRAD}_{\mathbf{x}}\left[N^{A}\left(\mathbf{x}_{n}^{p t}\right)\right]} \Omega_{0}^{p t} \\
& =\sum_{A=1}^{N_{\text {nodes }}} \mathbf{v}_{A, n+1 / 2} \Delta \mathrm{t} \cdot \sum_{p t=1}^{N_{p t}}\left(\mathbf{F}_{n+1 / 2}^{p t} \mathbf{S}^{p t}\right)\left(\mathbf{F}_{n}^{p t}\right)^{T} \operatorname{grad}_{n}\left[N^{A}\left(\mathbf{x}_{n}^{p t}\right)\right] \Omega_{0}^{p t} \\
& =\Delta \mathrm{t} \sum_{A=1}^{N_{\text {nodes }}} \mathbf{v}_{A, n+1 / 2} \cdot\left(\mathbf{F}^{i n t}\right)_{n+1 / 2}^{A} \text {. }
\end{aligned}
$$

Finally, consideration of equation $(29)_{1}$ yields

$$
\begin{aligned}
0 & =\sum_{A=1}^{N_{\text {Nodes }}} \mathbf{v}_{A, n+1 / 2} \cdot\left[\frac{1}{\Delta \mathrm{t}} \sum_{B=1}^{N_{\text {Nodes }}} \widetilde{M}_{n}^{A B}\left(\mathbf{v}_{B, n+1}-\mathbf{v}_{B, n}\right)+\left(\mathbf{F}^{\text {int }}\right)_{n+1 / 2}^{A}\right] \\
& =\frac{1}{\Delta \mathrm{t}}\left(\mathrm{T}_{n+1}^{h}-\mathrm{T}_{n}^{h}\right)+\frac{1}{\Delta \mathrm{t}}\left(\mathrm{V}_{n+1}^{p t}-\mathrm{V}_{n}^{p t}\right) \\
& =\frac{1}{\Delta \mathrm{t}}\left(\mathrm{H}_{n+1}^{h}-\mathrm{H}_{n}^{h}\right) .
\end{aligned}
$$


Since $\Delta \mathrm{t}>0, \mathrm{H}_{n+1}^{h}=\mathrm{H}_{n}^{h}$, which is the desired result.

Thus, for energy conservation, the symmetric tensor $\mathbf{S}^{p t}$ must be defined to satisfy the discrete dissipation (in)equality (50). The original construction of $\mathbf{S}^{p t}$ for general models of hyperelasticity can be found in [40], and is elaborated upon in [30]. However, a more convenient form is from references [21], [22] and is given as

$$
\mathbf{S}^{p t}:=\overline{\mathbf{S}}+\left[\frac{2\left[W\left(\mathbf{C}_{n+1}^{p t}\right)-W\left(\mathbf{C}_{n}^{p t}\right)\right]-\overline{\mathbf{S}}: \Delta \mathbf{C}^{p t}}{\left\|\Delta \mathbf{C}^{p t}\right\|^{2}}\right] \Delta \mathbf{C}^{p t}
$$

One may easily verify that $\frac{1}{2} \mathbf{S}^{p t}: \Delta \mathbf{C}^{p t}=W\left(\mathbf{C}_{n+1}^{p t}\right)-W\left(\mathbf{C}_{n}^{p t}\right)$ for any choice of $\overline{\mathbf{S}}$. The stress $\overline{\mathbf{S}}$ should be defined consistent with a midpoint approximation to the continuum stress formula (9) for second order accuracy. Said choice for $\overline{\mathbf{S}}$ is not unique, as noted in Remark 4.1 of [3]. However, there would appear to be at least three reasonable options:

1. stress at the configurational midpoint:

$$
\overline{\mathbf{S}}=\hat{\mathbf{S}}\left(\mathbf{F}_{n+1 / 2}^{T} \mathbf{F}_{n+1 / 2}\right) .
$$

2. stress at average strain:

$$
\overline{\mathbf{S}}=\hat{\mathbf{S}}\left(\frac{\mathbf{C}_{n}+\mathbf{C}_{n+1}}{2}\right) .
$$

This form is recommended in reference [21] and used in reference [3].

3. average stress:

$$
\overline{\mathbf{S}}=\frac{1}{2}\left[\hat{\mathbf{S}}\left(\mathbf{C}_{n}\right)+\hat{\mathbf{S}}\left(\mathbf{C}_{n+1}\right)\right] .
$$

A Taylor series expansion about $\mathbf{C}_{n}^{p t}$ provides some insight into these choices:

$$
\begin{aligned}
\mathbf{S}^{p t} & =\overline{\mathbf{S}}+\left[\frac{\left[\hat{\mathbf{S}}\left(\mathbf{C}_{n}^{p t}\right): \Delta \mathbf{C}^{p t}+\mathcal{O}\left(\left\|\Delta \mathbf{C}^{p t}\right\|^{2}\right)\right]-\overline{\mathbf{S}}: \Delta \mathbf{C}^{p t}}{\left\|\Delta \mathbf{C}^{p t}\right\|^{2}}\right] \Delta \mathbf{C}^{p t} \\
& =\overline{\mathbf{S}}+\left[\frac{\left(\hat{\mathbf{S}}\left(\mathbf{C}_{n}^{p t}\right)-\overline{\mathbf{S}}\right): \Delta \mathbf{C}^{p t}+\mathcal{O}\left(\left\|\Delta \mathbf{C}^{p t}\right\|^{2}\right)}{\left\|\Delta \mathbf{C}^{p t}\right\|^{2}}\right] \Delta \mathbf{C}^{p t} \\
& =\overline{\mathbf{S}}+\left[\frac{\left(\hat{\mathbf{S}}\left(\mathbf{C}_{n}^{p t}\right)-\overline{\mathbf{S}}\right): \Delta \mathbf{C}^{p t}}{\left\|\Delta \mathbf{C}^{p t}\right\|^{2}} \Delta \mathbf{C}^{p t}\right]+\mathcal{O}\left(\left\|\Delta \mathbf{C}^{p t}\right\|\right),
\end{aligned}
$$

so that

$$
\left\|\mathbf{S}^{p t}-\overline{\mathbf{S}}\right\| \leq\left\|\overline{\mathbf{S}}-\hat{\mathbf{S}}\left(\mathbf{C}_{n}^{p t}\right)\right\|+\mathcal{O}\left(\left\|\Delta \mathbf{C}^{p t}\right\|\right) .
$$

A sufficient condition to have a well defined limit as $\left\|\Delta \mathbf{C}^{p t}\right\| \searrow 0$ is

$$
\lim _{\left\|\Delta \mathbf{C}^{p t}\right\| \backslash 0} \overline{\mathbf{S}}=\hat{\mathbf{S}}\left(\mathbf{C}_{n}^{p t}\right) \Longrightarrow \lim _{\left\|\Delta \mathbf{C}^{p t}\right\| \backslash 0} \mathbf{S}^{p t}=\overline{\mathbf{S}}=\hat{\mathbf{S}}\left(\mathbf{C}_{n}^{p t}\right) .
$$


Thus choices (2) or (3) above are suitable. The limit behavior of choice (1) as $\| \Delta \mathbf{C} \searrow\rangle$ is unclear, since under a superposed rigid body motion $\mathbf{F}_{n+1}=\mathbf{Q F}_{n}$ for some $\mathbf{Q} \in S O(3)$, $\mathbf{C}_{n+1}=\mathbf{C}_{n}$ but

$$
\mathbf{F}_{n+1 / 2}=\frac{1}{2}\left(\mathbf{F}_{n}+\mathbf{Q} \mathbf{F}_{n}\right) \Longrightarrow \mathbf{F}_{n+1 / 2}^{T} \mathbf{F}_{n+1 / 2}=\frac{1}{2} \mathbf{C}_{n}+\frac{1}{4}\left[\mathbf{F}_{n}^{T}\left(\mathbf{Q}+\mathbf{Q}^{T}\right) \mathbf{F}_{n}\right] \neq \mathbf{C}_{n} .
$$

This observation is consistent with the discussion in [21], where it is noted that the use of the configurational midpoint strain introduces an artificial coupling between rigid rotations and material stretches which is not present in the continuum problem. Note, however, that the limit behavior of choice (1) is well defined in the limit as $\|\Delta \mathbf{F}\| \searrow 0$. All the numerical simulations in this work use choice (2), the stress at average strain.

\section{Remarks 3.5.}

1. Notice that $\mathbf{S}^{p t}$ as defined in equation (54) is a rank one modification of the stress $\overline{\mathbf{S}}$. On the orthogonal complement of $\Delta \mathbf{C}^{p t}$ the two tensors are the same:

$$
\left(\mathbf{S}^{p t}-\overline{\mathbf{S}}\right): \mathbf{M}=0 \quad \forall \mathbf{M} \quad \text { such that } \quad \mathbf{M}: \Delta \mathbf{C}^{p t}=0 .
$$

Thus $\overline{\mathbf{S}}$ is modified only in the direction of $\Delta \mathbf{C}^{p t}$ to ensure that energy is conserved. In this regard the construction of $\mathbf{S}^{p t}$ from $\left\{\overline{\mathbf{S}}, W_{n}, W_{n+1}\right\}$ is directly analogous to the rank one Jacobian update of Broyden's method [14], [15]. In fact, the constrained (convex) minimization conditions

$$
\left.\begin{array}{l}
\widehat{\mathcal{T}}_{3 \times 3}^{\text {sym }}:=\left\{\mathbf{S} \in \mathcal{T}_{3 \times 3}^{\text {sym }} \mid \frac{1}{2} \mathbf{S}: \Delta \mathbf{C}^{p t}=W\left(\mathbf{C}_{n+1}^{p t}\right)-W\left(\mathbf{C}_{n}^{p t}\right)\right\} \\
\in \widehat{\mathcal{T}}_{3 \times 3}^{\text {sym }} \quad \text { such that }\left\|\mathbf{S}^{p t}-\overline{\mathbf{S}}\right\|^{2} \leq\left\|\mathbf{S}^{\prime}-\overline{\mathbf{S}}\right\|^{2} \quad \forall \mathbf{S}^{\prime} \in \widehat{\mathcal{T}}_{3 \times 3}^{\text {sym }}
\end{array}\right\},
$$

are sufficient to uniquely determine $\mathbf{S}^{p t}$ (reference [19], Theorem 4.1). The unique solution is, of course, given by (54), and the symmetric algorithmic stress $\mathbf{S}^{p t}$ is the closest tensor to $\overline{\mathbf{S}}$ which also satisfies equation (50). Equation (54) can also be interpreted as a discrete derivative (Definition 4.1 of [22]) of the stored energy function $W(\mathbf{C})$.

2. If the (non-zero) body force $\mathbf{b}$ is derivable from a potential function $\psi_{g}(\boldsymbol{\varphi})$ such that $\mathbf{b}=-\partial_{\boldsymbol{\varphi}} \psi_{g}$, then the body force term $\mathbf{b}_{n+1 / 2}$ in equation (30b) should be evaluated as a discrete derivative of $\psi_{g}$,

$$
-\mathbf{b}_{n+1 / 2}:=\partial_{\boldsymbol{\varphi}} \psi_{g}\left(\boldsymbol{\varphi}_{n+1 / 2}\right)+\left[\frac{\psi_{g}\left(\boldsymbol{\varphi}_{n+1}\right)-\psi_{g}\left(\boldsymbol{\varphi}_{n}\right)-\partial_{\boldsymbol{\varphi}} \psi_{g}\left(\boldsymbol{\varphi}_{n+1 / 2}\right) \cdot \Delta \boldsymbol{\varphi}}{\|\Delta \boldsymbol{\varphi}\|^{2}}\right] \Delta \boldsymbol{\varphi},
$$

to ensure energy conservation. For a linear gravitational potential $\psi_{g}(\boldsymbol{\varphi}):=-\mathbf{g} \cdot(\boldsymbol{\varphi}-$ $\mathbf{x}_{0}$ ), with $\mathbf{g}, \mathbf{x}_{0} \in \mathbb{R}^{3}$, the discrete derivative and the exact derivative coincide and $\mathbf{b}_{n+1 / 2}=\mathbf{g}$. The same argument holds for the (non-zero) tractions $\overline{\mathbf{T}}_{n+1 / 2}$ should they be derivable from a potential. 


\subsubsection{The material-point update}

The general observation of this section is that, with a consistent mass matrix, changes in momentum and energy computed on the grid are exactly reflected in equal changes in total momentum and energy for the material points. Therefore, if the grid solution, like the one above, is conservative, then so is MPM. The proofs below essentially follow the algebraic manipulations established in the references on FLIP. The energy analysis of FLIP appears in [12], [13] for the lumped mass matrix and in [16] for the consistent and effective mass matrices. Angular momentum is examined for FLIP in [11] where it is incorrectly concluded that the change in angular momentum on the grid and material points is equal when the lumped mass matrix is used. Reference [16] cites [11] for the lumped mass matrix, but correctly states that a consistent mass matrix yields equal changes in angular momentum on the grid and material points. However, the comment in the Appendix of [16] which states that the effective masses also have this property is incorrect.

Proposition 3.8. The material-point update conserves the increment in total linear momentum, $\Delta \mathbf{L}^{p t}=\Delta \mathbf{L}^{h}$.

Proof. This proof is simple algebra:

$$
\begin{aligned}
\Delta \mathbf{L}^{p t} & =\mathbf{L}_{n+1}^{p t}-\mathbf{L}_{n}^{p t} \\
& =\sum_{p t=1}^{N_{p t}} m^{p t}\left(\mathbf{v}_{n+1}^{p t}-\mathbf{v}_{n}^{p t}\right) \\
& =\sum_{p t=1}^{N_{p t}} m^{p t} \Delta \mathbf{v}^{h}\left(\mathbf{x}_{n}^{p t}\right) \quad\left(\text { by }(32)_{2}\right) \\
& =\sum_{p t=1}^{N_{p t}}\left(\sum_{A=1}^{N_{n o d e s}} m^{p t} N^{A}\left(\mathbf{x}_{n}^{p t}\right) \Delta \mathbf{v}_{A}\right) \\
& =\sum_{A=1}^{N_{n o d e s}}\left(\sum_{p t=1}^{N_{p t}} m^{p t} N^{A}\left(\mathbf{x}_{n}^{p t}\right)\right) \Delta \mathbf{v}_{A} \\
& =\sum_{A=1}^{N_{n o d e s}} \bar{M}_{n}^{A A} \Delta \mathbf{v}_{A} \quad(\text { by }(25)) \\
& =\mathbf{L}_{n+1}^{h}-\mathbf{L}_{n}^{h} \quad(\text { by Definition 3.1) } \\
& =\Delta \mathbf{L}^{h} .
\end{aligned}
$$

For the last step, recall that the mass matrix is constant over the time step $\left[\mathrm{t}_{n}, \mathrm{t}_{n+1}\right]$.

Corollary 3.9. Assume $\left(\mathbf{F}^{e x t}\right)_{n+1 / 2}^{A}=\mathbf{0}$. Then Algorithm MPM conserves exactly the total material-point linear momentum.

Proof. Combine Propositions 3.5 and 3.8. 
Proposition 3.10. During the material-point update, the increment in total material-point angular momentum is

$$
\Delta \mathbf{J}^{p t}=\Delta \mathbf{J}^{h}-\varepsilon\left[\sum_{A, B=1}^{N_{\text {nodes }}} \boldsymbol{\varphi}_{A, n+1} \times\left(\bar{M}_{n}^{A B}-M_{n}^{A B}\right) \Delta \mathbf{v}_{B}\right] .
$$

Proof. Start with the definition of $\mathbf{J}_{n+1}^{p t}$ and recall Proposition 3.3, which shows that $\mathbf{J}_{n}^{h}=\mathbf{J}_{n}^{p t}$, to obtain

$$
\begin{aligned}
\mathbf{J}_{n+1}^{p t} & =\sum_{p t=1}^{N_{p t}} \boldsymbol{\varphi}_{n+1}^{p t} \times m^{p t} \mathbf{v}_{n+1}^{p t} \\
& =\sum_{p t=1}^{N_{p t}} \boldsymbol{\varphi}_{n+1}^{p t} \times m^{p t}\left(\mathbf{v}_{n}^{p t}+\Delta \mathbf{v}^{h}\left(\mathbf{x}_{n}^{p t}\right)\right) \quad\left(\text { by }(32)_{2}\right) \\
& =\sum_{p t=1}^{N_{p t}}\left(\sum_{A=1}^{N_{\text {nodes }}} N^{A}\left(\mathbf{x}_{n}^{p t}\right) \boldsymbol{\varphi}_{A, n+1}\right) \times m^{p t}\left(\mathbf{v}_{n}^{p t}+\left(\sum_{B=1}^{N_{\text {nodes }}} N^{B}\left(\mathbf{x}_{n}^{p t}\right) \Delta \mathbf{v}_{B}\right)\right) \\
& =\sum_{A=1}^{N_{\text {nodes }}} \boldsymbol{\varphi}_{A, n+1} \times\left[\sum_{p t=1}^{N_{p t}} N^{A}\left(\mathbf{x}_{n}^{p t}\right)\left(m^{p t} \mathbf{v}_{n}^{p t}+\sum_{B=1}^{N_{\text {nodes }}} m^{p t} N^{B}\left(\mathbf{x}_{n}^{p t}\right) \Delta \mathbf{v}_{B}\right)\right] \\
& =\sum_{A=1}^{N_{\text {nodes }}} \boldsymbol{\varphi}_{A, n+1} \times\left[\sum_{B=1}^{N_{\text {nodes }}} \widetilde{M}_{n}^{A B} \mathbf{v}_{B, n}+\sum_{p t=1}^{N_{p t}} \sum_{B=1}^{N_{\text {nodes }}} m^{p t} N^{A}\left(\mathbf{x}_{n}^{p t}\right) N^{B}\left(\mathbf{x}_{n}^{p t}\right) \Delta \mathbf{v}_{B}\right] \quad(\mathrm{by}(28)) \\
& =\sum_{A=1}^{N_{\text {nodes }}} \boldsymbol{\varphi}_{A, n+1} \times\left[\sum_{B=1}^{N_{\text {nodes }}} \widetilde{M}_{n}^{A B}\left(\mathbf{v}_{B, n+1}-\Delta \mathbf{v}_{B}\right)+\sum_{p t=1}^{N_{p t}} \sum_{B=1}^{N_{\text {nodes }}} m^{p t} N^{A}\left(\mathbf{x}_{n}^{p t}\right) N^{B}\left(\mathbf{x}_{n}^{p t}\right) \Delta \mathbf{v}_{B}\right] \\
& =\mathbf{J}_{n}^{h}+\Delta \mathbf{J}^{h}+\sum_{A, B=1}^{N_{\text {nodes }}} \boldsymbol{\varphi}_{A, n+1} \times\left(M_{n}^{A B}-\widetilde{M}_{n}^{A B}\right) \Delta \mathbf{v}_{B} \\
& =\mathbf{J}_{n}^{p t}+\Delta \mathbf{J}^{h}-\varepsilon \sum_{A, B=1}^{N_{\text {nodes }}} \boldsymbol{\varphi}_{A, n+1} \times\left(\bar{M}_{n}^{A B}-M_{n}^{A B}\right) \Delta \mathbf{v}_{B}\left(b_{y}(26)\right) .
\end{aligned}
$$

This equation is the desired relation for $\Delta \mathbf{J}^{p t}$.

Corollary 3.11. Assume $\left(\mathbf{F}^{e x t}\right)_{n+1 / 2}^{A}=\mathbf{0}$. Then, for Algorithm MPM, the total materialpoint angular momentum evolves as:

$$
\mathbf{J}_{n+1}^{p t}=\mathbf{J}_{n}^{p t}-\varepsilon\left[\sum_{A, B=1}^{N_{\text {nodes }}} \boldsymbol{\varphi}_{A, n+1} \times\left(\bar{M}_{n}^{A B}-M_{n}^{A B}\right) \Delta \mathbf{v}_{B}\right] .
$$

In particular, if $\varepsilon=0$, then Algorithm MPM conserves exactly the total material-point angular momentum.

Proof. Combine Propositions 3.6 and 3.10. 
Proposition 3.12. During the material-point update, the increment in total material-point kinetic energy is

$$
\Delta \mathrm{T}^{p t}=\Delta \mathrm{T}^{h}-\frac{1}{2} \varepsilon\left[\Delta \mathbf{v}^{h} \cdot\left(\overline{\mathbf{M}}_{n}-\mathbf{M}_{n}\right) \Delta \mathbf{v}^{h}\right]
$$

which implies that $\Delta \mathrm{T}^{p t} \leq \Delta \mathrm{T}^{h}$ by Lemma 3.1 .

Proof. Again, this proof is purely algebraic.

$$
\begin{aligned}
& 2 \Delta \mathrm{T}^{p t}=2\left(\mathrm{~T}_{n+1}^{p t}-\mathrm{T}_{n}^{p t}\right) \\
& =\sum_{p t=1}^{N_{p t}} m^{p t}\left(\left\|\mathbf{v}_{n+1}^{p t}\right\|^{2}-\left\|\mathbf{v}_{n}^{p t}\right\|^{2}\right) \\
& =\sum_{p t=1}^{N_{p t}} m^{p t}\left(\mathbf{v}_{n+1}^{p t}-\mathbf{v}_{n}^{p t}\right) \cdot\left(\mathbf{v}_{n}^{p t}+\mathbf{v}_{n+1}^{p t}\right) \\
& =\sum_{p t=1}^{N_{p t}} m^{p t} \Delta \mathbf{v}^{h}\left(\mathbf{x}_{n}^{p t}\right) \cdot\left(2 \mathbf{v}_{n}^{p t}+\Delta \mathbf{v}^{h}\left(\mathbf{x}_{n}^{p t}\right)\right) \quad\left(\text { by }(32)_{2}\right) \\
& =\sum_{A=1}^{N_{\text {nodes }}}\left(2 \sum_{p t=1}^{N_{p t}} N^{A}\left(\mathbf{x}_{n}^{p t}\right) m^{p t} \mathbf{v}_{n}^{p t}\right) \cdot \Delta \mathbf{v}_{A}+\sum_{A, B=1}^{N_{\text {nodes }}}\left(\sum_{p t=1}^{N_{p t}} m^{p t} N^{A}\left(\mathbf{x}_{n}^{p t}\right) N^{B}\left(\mathbf{x}_{n}^{p t}\right)\right) \Delta \mathbf{v}_{A} \cdot \Delta \mathbf{v}_{B} \\
& =\sum_{A, B=1}^{N_{\text {nodes }}}\left(2 \widetilde{M}_{n}^{A B} \mathbf{v}_{B, n} \cdot \Delta \mathbf{v}_{A}+\Delta \mathbf{v}_{A} \cdot M_{n}^{A B} \Delta \mathbf{v}_{B}\right) \quad(\text { by }(28)) \\
& =2 \mathbf{v}_{n}^{h} \cdot \widetilde{\mathbf{M}}_{n} \Delta \mathbf{v}^{h}+\Delta \mathbf{v}^{h} \cdot \mathbf{M}_{n} \Delta \mathbf{v}^{h} \\
& =2 \mathbf{v}_{n}^{h} \cdot \widetilde{\mathbf{M}}_{n} \Delta \mathbf{v}^{h}+\Delta \mathbf{v}^{h} \cdot\left(\mathbf{M}_{n}-\widetilde{\mathbf{M}}_{n}\right) \Delta \mathbf{v}^{h}+\Delta \mathbf{v}^{h} \cdot \widetilde{\mathbf{M}}_{n} \Delta \mathbf{v}^{h} \\
& =\left(2 \mathbf{v}_{n}^{h}+\Delta \mathbf{v}^{h}\right) \cdot \widetilde{\mathbf{M}}_{n} \Delta \mathbf{v}^{h}+\Delta \mathbf{v}^{h} \cdot\left(\mathbf{M}_{n}-\widetilde{\mathbf{M}}_{n}\right) \Delta \mathbf{v}^{h} \\
& =\left(\mathbf{v}_{n}^{h}+\mathbf{v}_{n+1}^{h}\right) \cdot \widetilde{\mathbf{M}}_{n}\left(\mathbf{v}_{n}^{h}-\mathbf{v}_{n+1}^{h}\right)+\Delta \mathbf{v}^{h} \cdot\left(\mathbf{M}_{n}-\widetilde{\mathbf{M}}_{n}\right) \Delta \mathbf{v}^{h} \\
& =2\left(\mathrm{~T}_{n+1}^{h}-\mathrm{T}_{n}^{h}\right)+\Delta \mathbf{v}^{h} \cdot\left(\mathbf{M}_{n}-\widetilde{\mathbf{M}}_{n}\right) \Delta \mathbf{v}^{h} \\
& =2 \Delta \mathrm{T}^{h}-\varepsilon \underbrace{\left[\Delta \mathbf{v}^{h} \cdot\left(\overline{\mathbf{M}}_{n}-\mathbf{M}_{n}\right) \Delta \mathbf{v}^{h}\right]}_{\geq 0} \\
& \leq 2 \Delta \top^{h}
\end{aligned}
$$

Corollary 3.13. Assume $\left(\mathbf{F}^{e x t}\right)_{n+1 / 2}^{A}=\mathbf{0}$ and $\mathbf{S}^{p t}$ satisfies equation (50). Then, for Algorithm MPM, the total material-point energy evolves as:

$$
\mathrm{H}_{n+1}^{p t}=\mathrm{H}_{n}^{p t}-\frac{1}{2} \varepsilon\left[\Delta \mathbf{v}^{h} \cdot\left(\overline{\mathbf{M}}_{n}-\mathbf{M}_{n}\right) \Delta \mathbf{v}^{h}\right]
$$

In particular, this implies that $\mathrm{H}_{n+1}^{p t} \leq \mathrm{H}_{n}^{p t}$ so that Algorithm MPM is unconditionally stable, and exactly energy conserving for $\varepsilon=0$. 
Proof. Combining Propositions 3.7 and 3.12 yields

$$
\begin{aligned}
\mathrm{H}_{n+1}^{p t} & =\mathrm{H}_{n}^{p t}+\Delta \mathrm{H}^{p t} \\
& =\mathrm{H}_{n}^{p t}+\left(\Delta \mathrm{T}^{p t}+\Delta \mathrm{V}^{p t}\right) \\
& =\mathrm{H}_{n}^{p t}+\left(\Delta \mathrm{T}^{h}+\Delta \mathrm{V}^{p t}\right)-\frac{1}{2} \varepsilon\left[\Delta \mathbf{v}^{h} \cdot\left(\overline{\mathbf{M}}_{n}-\mathbf{M}_{n}\right) \Delta \mathbf{v}^{h}\right] \\
& =\mathrm{H}_{n}^{p t}+\Delta \mathrm{H}^{h}-\frac{1}{2} \varepsilon\left[\Delta \mathbf{v}^{h} \cdot\left(\overline{\mathbf{M}}_{n}-\mathbf{M}_{n}\right) \Delta \mathbf{v}^{h}\right] \\
& =\mathbf{H}_{n}^{p t}-\frac{1}{2} \varepsilon \underbrace{\left[\Delta \mathbf{v}^{h} \cdot\left(\overline{\mathbf{M}}_{n}-\mathbf{M}_{n}\right) \Delta \mathbf{v}^{h}\right]}_{\geq 0} \\
& \leq \mathbf{H}_{n}^{p t} .
\end{aligned}
$$

\section{Remarks 3.6.}

1. Given the above conservation properties, the most important observation to make is that Algorithm MPM directly inherits the conservation properties of the underlying Lagrangian finite element time integrator, if the consistent mass matrix is used. The choice of this time-stepping scheme is a fundamental step in the construction of a material-point algorithm. Use of a lumped mass matrix still conserves linear momentum but causes an $\mathcal{O}(h \Delta t)$ error in angular momentum conservation. Similarly, energy is dissipated with a lumped-mass formulation, but is conserved exactly using consistent mass. The dissipation is $\mathcal{O}\left(h \Delta t^{2}\right)$.

2. Even for a standard Lagrangian finite element simulation, there may be multiple measures of angular momentum and energy. If the analysis uses a lumped mass matrix, then the conserved quantities are the angular momentum and energy computed using that matrix. The corresponding quantities evaluated by Gaussian integration over the elements (which are the consistent mass quantities) are generally not conserved. However, one would expect those values to closely shadow the evolution of the lumped mass quantities. For a finite dimensional system the lumped and consistent mass matrices induce equivalent norms on the velocity field ${ }^{1}$, so that stability with respect to the lumped norm implies stability with respect to the consistent norm (and vice versa).

3. It is not necessary to use a midpoint-based energy conserving integrator for step 2 of Algorithm MPM:

(a) Most work in MPM to date has used an explicit time integrator with lumpedmass and a hypoelastic formulation [46]. Although not always explicitly indicated, the time stepping is typically based on a leapfrog method where the displacement, strain and stress are staggered by a half step in time compared to the velocity [25]. The algebraic techniques used to monitor conservation properties are the same as those used in this paper for the material-point-to-grid projection and the grid-tomaterial-point update. Only the analysis of the grid dynamics differs. For explicit

\footnotetext{
${ }^{1}$ All norms on a given finite dimensional (real) vector space are equivalent.
} 
grid dynamics, stability is usually conditional and the time step is bounded by the CFL number. The CFL number is based on the mesh size and wave speed in the material. Hence stability of an explicit MPM algorithm depends on the background mesh spacing and not on the distance between material points.

(b) Reference [18] contains an implicit MPM method with lumped mass and a smallstrain hypoelastic constitutive model. The time differencing is similar to what is used in this paper. In reference [43] an implicit integrator of the form

$$
\left.\begin{array}{r}
\frac{1}{\Delta \mathrm{t}} \overline{\mathbf{M}}_{n}\left(\mathbf{v}_{n+1}^{h}-\mathbf{v}_{n}^{h}\right)+\left[(1-\alpha) \mathbf{F}_{n}^{i n t}+\alpha \mathbf{F}_{n+1}^{i n t}\right]=\mathbf{0} \\
\frac{1}{\Delta \mathrm{t}} \Delta \boldsymbol{\varphi}^{h}=\mathbf{v}_{n+1}^{h}
\end{array}\right\},
$$

is used instead. For linear problems, this method is unconditionally stable if and only if $\alpha=1$ (see [29]). Neither approach conserves energy or angular momentum. An alternative approach would be to use the implicit, momentum and energy conserving time integrator designed for hypoelastic models [36] combined with MPM.

(c) In reference [23] the authors consider hyperelasticity using the trapezoidal rule, a specific type of Newmark-Beta algorithm [26], along with a lumped mass approximation. Numerical experiments have shown that the trapezoidal rule is only conditionally stable for nonlinear problems [40]. However, given a sufficiently small time step, the trapezoidal rule possesses remarkable stability and conservation properties which stem from the discrete variational character of the scheme [28], [32]. It can also be shown that the trapezoidal rule conserves the midpoint angular momentum, i.e. $\mathbf{J}_{n+1 / 2}^{h}=\mathbf{J}_{n-1 / 2}^{h}[41]$. However, this result does not extend to an MPM algorithm where the mass matrix is only constant within a time step, not across time steps.

4. Unfortunately, a consistent linearization of equations (29) - (31), with the stress defined as in (54), produces an unsymmetric tangent matrix [21], [30], [40].

\section{Numerical simulations}

The goal of this section is to present some relatively simple numerical simulations which demonstrate the conservation properties of Algorithm MPM. All numerical simulations in this work use a nonlinear quasi-Newton root finding algorithm coupled with a matrix-free iterative GMRES linear equation solving algorithm. These are provided by the computational library PETSC [4], [5], [6]. Additionally, all simulations use standard displacement-based isoparametric four-node quadrilateral finite elements.

For all numerical simulations a compressible Neo-Hookean material model is used. The 
model takes the form

$$
\left.\begin{array}{r}
\tilde{\mathbf{C}}:=(\operatorname{det} \mathbf{C})^{-1 / 3} \mathbf{C} \\
W(\mathbf{C}):=\frac{1}{2} \mu[\operatorname{trace}(\tilde{\mathbf{C}})-3]+\kappa U\left[(\operatorname{det} \mathbf{C})^{1 / 2}\right] \\
U(J):=\frac{1}{4}\left[\left(J^{2}-1\right)-2 \log J\right]
\end{array}\right\},
$$

with $\mu>0$ and $\kappa>0$. The material parameters $\mu$ and $\kappa$ are the (linearized) shear modulus and (linearized) bulk modulus, respectively. This model is both strongly elliptic and polyconvex and is commonly used in the finite element simulation of rubber-like materials.

\subsection{Rebound of an elastic cylinder}

The first numerical example involves the (plane strain) impact and rebound of an elastic cylinder off rigid, frictionless walls. The cylinder is constrained to move within a box of dimension $15.0 \times 5$.0. The cylinder has an initial radius of 1.5 and is initially positioned at $\{2.5,2.5\}$. The initial velocity is $\{+0.5,0.0\}$. The density is $\rho_{0}=4.0$ and the elastic constants are $\mu=30.0$ and $\kappa=170.0$. The time step is set to $\Delta \mathrm{t}=0.05$. The background mesh consists of $30 \times 10$ elements. The nodal boundary conditions are prescribed such that on the boundaries where $x_{1}=0.0$ and $x_{1}=15.0$ the normal displacement (and thus velocity) is constrained to be zero (0.0).

Figure 2 shows the sequence of deformed particle configurations for a consistent mass simulation. The initial configuration is shown in Fig. 2(a). The cylinder translates to the right (Fig. 2(b)), impacts the wall (Fig. 2(c)), and rebounds (Fig. 2(d)). After translating to the left, the cylinder impacts the left boundary (Fig. 2(e)), and rebounds (Fig. 2(f)). Translation to the right, and another impact and rebound from the right boundary are shown in Figs. 2(g-j). The initial impact velocity is $8 \%$ of the linearized bulk wave speed. This velocity is large enough to make the deformation of the cylinder visible upon impact.

Figure $3(\mathrm{a})$ is a plot of the energy evolution for a consistent mass calculation. Total energy is shown with a solid line, potential energy is indicated with a dotted line and kinetic energy is shown with a dashed line. The energy consists of only kinetic energy up to the time of the first impact since the cylinder is undergoing rigid translation. During each impact, the kinetic energy drops and then rises. Not all of the kinetic energy prior to impact is recovered after impact since some energy is converted to potential energy, which manifests itself as ringing of the cylinder while it translates. Each impact increases the ringing. The contact time can be approximated by the amount of time it takes for a wave to propagate across the body and back. For this purpose, the initial diameter of the body and the linearized bulk wave speed are used to estimate a contact time of $T_{c}=0.92$. The contact time is about 18 times the time step, so reasonable accuracy is expected.

A careful examination of Fig. 3(a) shows that there is a slight decrease in the total energy over the course of the simulation. This small decrease in energy is due to the error tolerance on the nonlinear Newton solver. Default values for the convergence criteria were used with the PETSC library [4], [5], [6]. The option to use the Eisenstat-Walker convergence criterion for the linear solver was invoked [20], again with default values. It is possible that the error 


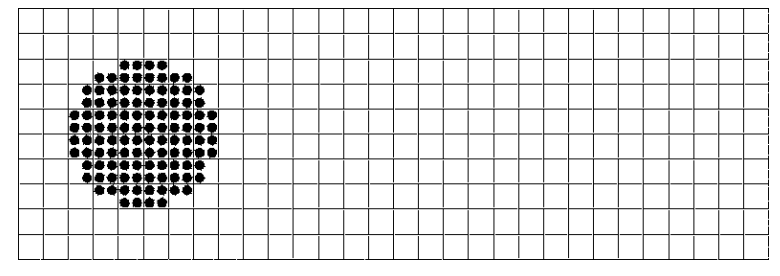

(a) Time $t=0.0$

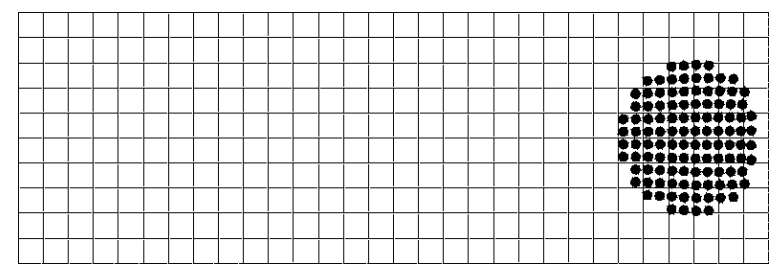

(c) Time $t=22.0$

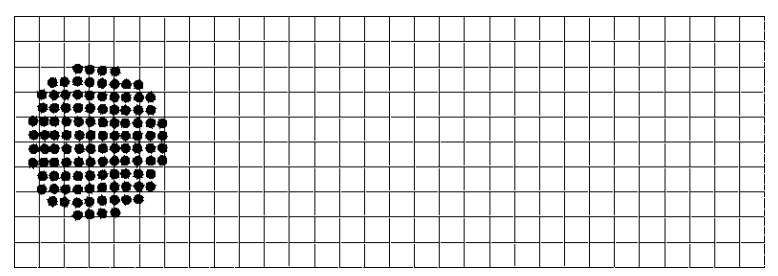

(e) Time $\mathrm{t}=48.0$

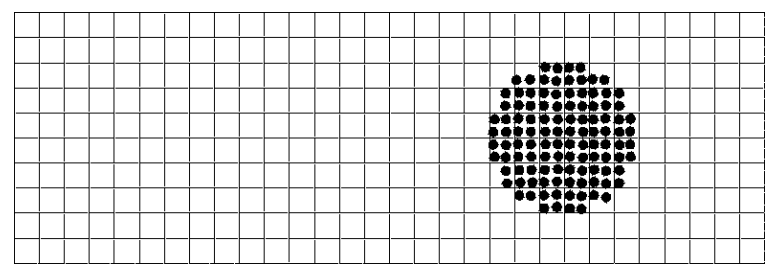

(g) Time $\mathrm{t}=70.0$

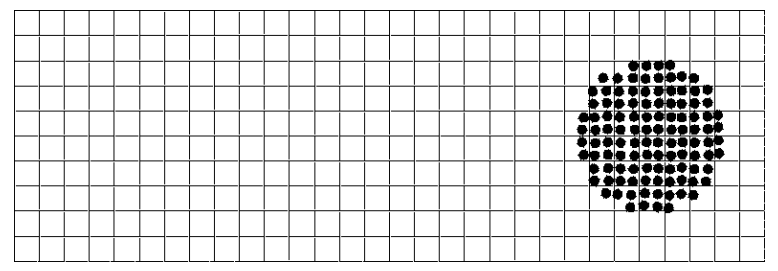

(i) Time $\mathrm{t}=78.0$

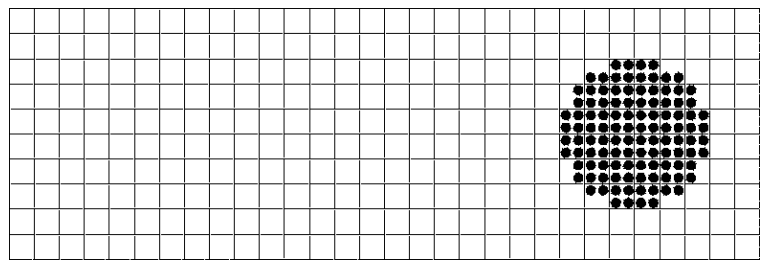

(b) Time $t=20.0$

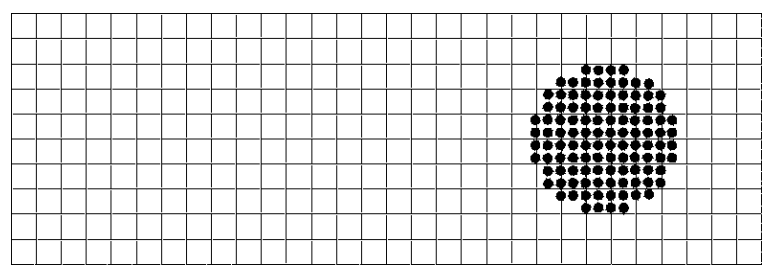

(d) Time $t=26.0$

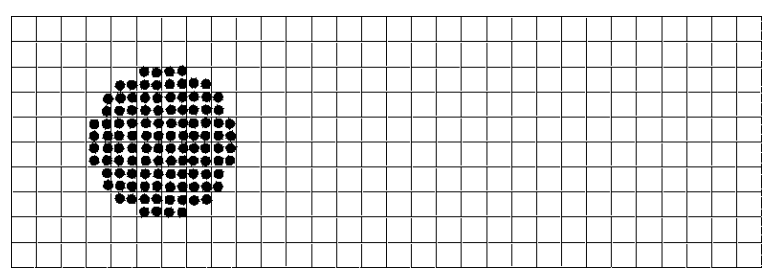

(f) Time $t=52.0$

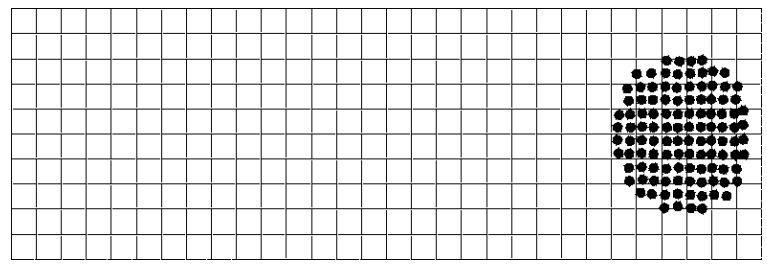

(h) Time $t=76.0$

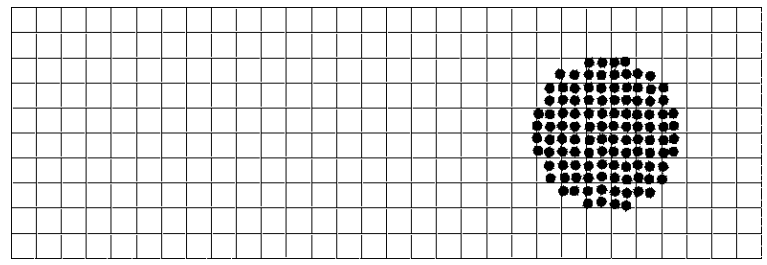

(j) Time $t=80.0$

Figure 2: Rebound of an elastic cylinder. Sequence of deformed particle configurations for a consistent mass simulation. 


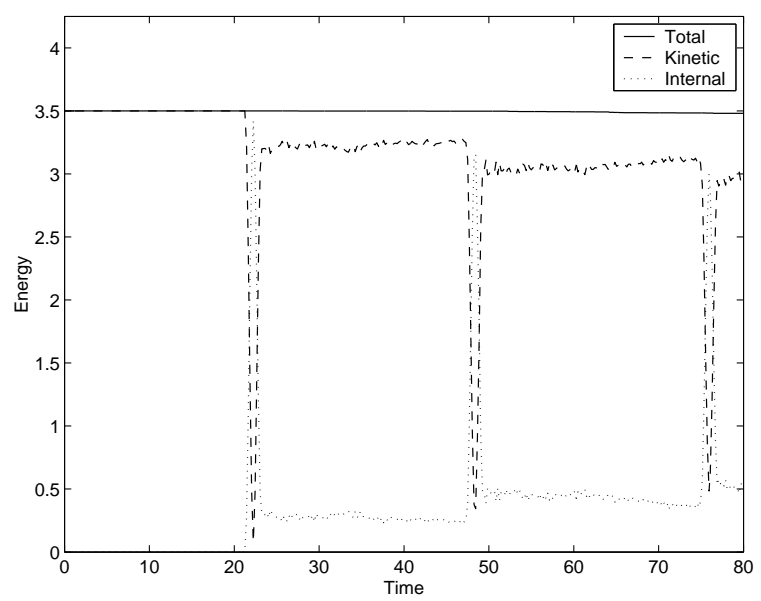

(a) Consistent Mass

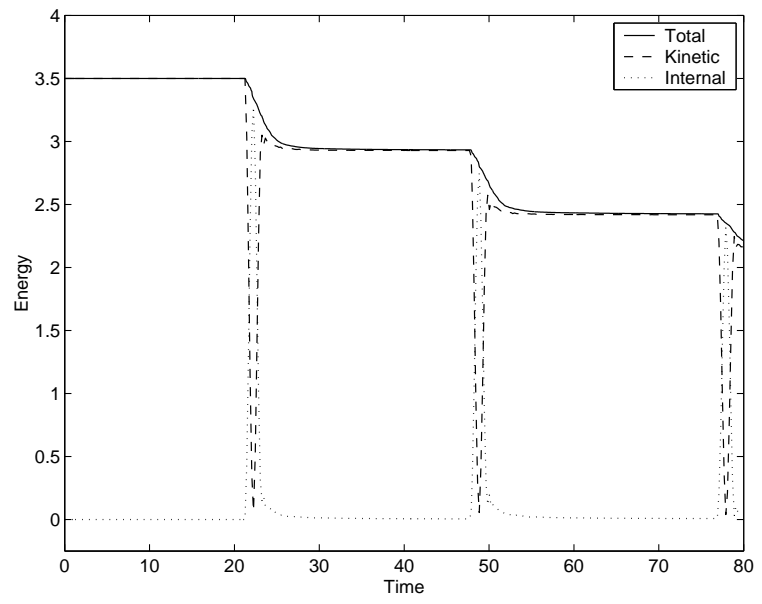

(b) Lumped Mass

Figure 3: Rebound of an elastic cylinder. (a) Evolution of total, kinetic and internal energies for a consistent mass calculation. The total energy is conserved. (b) Evolution of total, kinetic and internal energies for a lumped mass calculation.

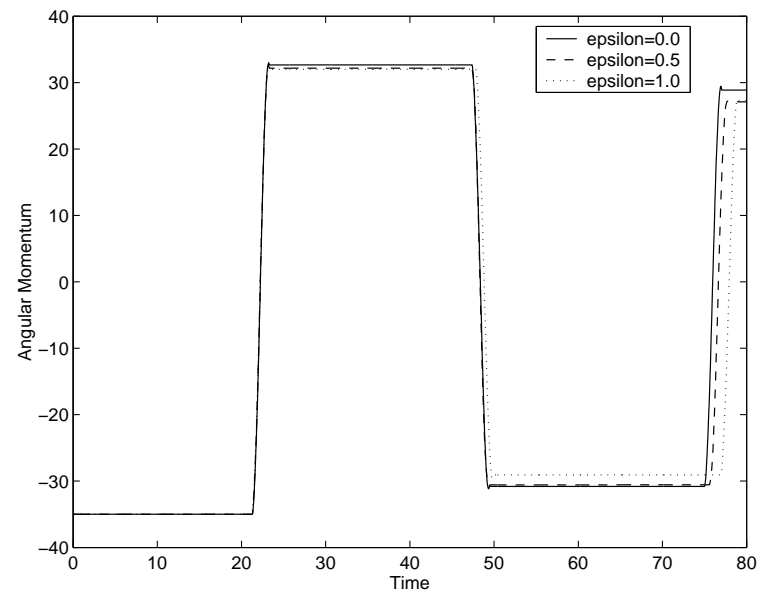

(a) Angular Momentum

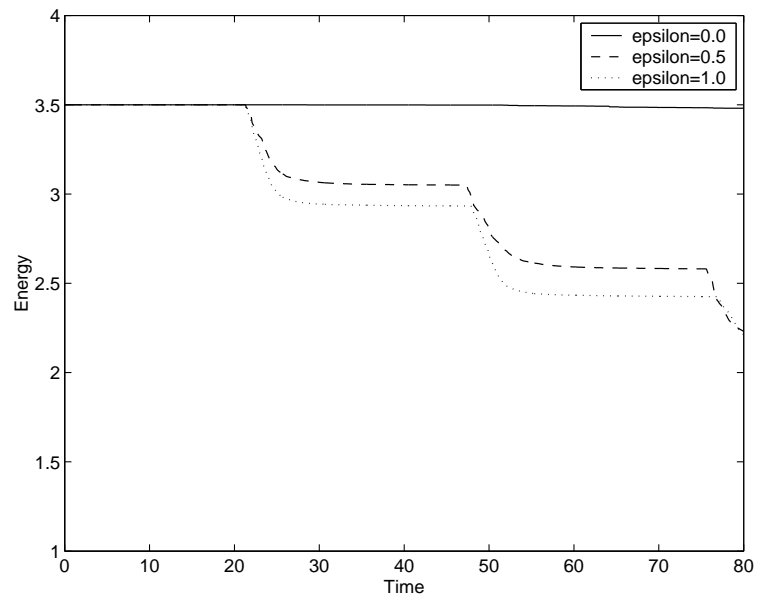

(b) Energy

Figure 4: Rebound of an elastic cylinder. (a) Evolution of angular momentum for three different choices of the parameter $\varepsilon$. (b) Evolution of the total energy for three different choices of the parameter $\varepsilon$. As expected, when $\varepsilon>0$ the method is dissipative. 
tolerance was not exactly satisfied on some steps and iteration ceased due to a limit on the number of function evaluations.

Figure 4(a) shows the evolution of angular momentum. The solid line is for the consistent mass simulation $(\varepsilon=0.0)$. Angular momentum changes only due to impact with the boundaries of the domain. The change in sign of the angular momentum reflects the change in translation direction after each impact. Figure 4(b) also shows the result of a simulation using an effective mass matrix $(\varepsilon=0.5)$ and the lumped mass matrix $(\varepsilon=1.0)$. For this example, since the cylinder is not rotating, angular momentum does not vary much with $\varepsilon$. When $\varepsilon>0$ the magnitude of angular momentum is slightly reduced and there is a shift in where, in time, the impacts occur due to a reduced velocity for the cylinder when mass lumping is used. The reduced velocity is apparent also in Fig. 3(b) where the energy is plotted for a lumped mass calculation $(\varepsilon=1.0)$. Each impact of the cylinder dissipates energy. There is little energy dissipation during the intervals where the cylinder is translating.

For comparison, Figure 4(b) shows the total energy evolution for different choices of the parameter $\varepsilon$. Again, mass lumping dissipates energy noticeably during impact, and only slightly during translation with ringing. The lumped mass matrix, $\epsilon=1.0$, tends to dissipate more energy than the effective mass matrix with $\epsilon=0.5$, although this is not guaranteed. The discussion of the previous sections shows that for the same velocity field, increasing $\varepsilon$ increases the dissipation. However, the mass matrix affects the dynamics and the velocity fields differ for simulations with different $\varepsilon$.

\subsection{Skew impact of two elastic cylinders}

This example involves the (plane strain) skew impact of two elastic cylinders. The cylinders are positioned to move within a box of dimension $20.0 \times 12.0$. The cylinders have an initial radius of 2.0 and are initially centered at $\{3.0,3.0\}$ and $\{16.0,5.0\}$. The initial velocities are $\{+0.75,0.0\}$ and $\{-0.75,0.0\}$, respectively. For both cylinders the density is $\rho_{0}=5.0$ and the elastic constants are $\mu=11.0$ and $\kappa=81.0$. The time step is set to $\Delta \mathrm{t}=0.333$. The background mesh consists of $20 \times 12$ elements. This problem is similar to the second numerical example in Section 4.2 of [2]. The PETSC parameters restrict the number of nonlinear iterations to 20 with a relative error tolerance of $10^{-8}$. The linear solver is allowed a maximum of 500 iterations and a relative error tolerance of $10^{-4}$.

Figure 5 shows the sequence of deformed particle configurations for a consistent mass simulation. The initial configuration is shown in Fig. 5(a). The cylinders translate towards each other (Fig. 5(b)). The impact is skew (Fig. 5(c-d)). Figs. 5(e-h) show the rebound and subsequent separation of the cylinders. In this simulation, the initial relative impact velocity is $37 \%$ of the linearized bulk wave speed, and the contact time is $T_{c}=0.99$, about $3 \Delta t$. The high impact velocity results in large deformation of the cylinders and substantial ringing after impact. Figure 6 is a plot of the energy evolution for this simulation. The solid line is the total energy, which is conserved. The dotted line with square markers indicates the kinetic energy as a function of time. Initially all energy is kinetic but after impact, some of the kinetic energy is converted to elastic potential energy. The potential energy is shown with a dotted line marked by circles. The oscillations in the kinetic and potential energies after impact are indicative of the ringing of the cylinders.

Figure 7 (a) shows the evolution of angular momentum. The solid line shows that angular 


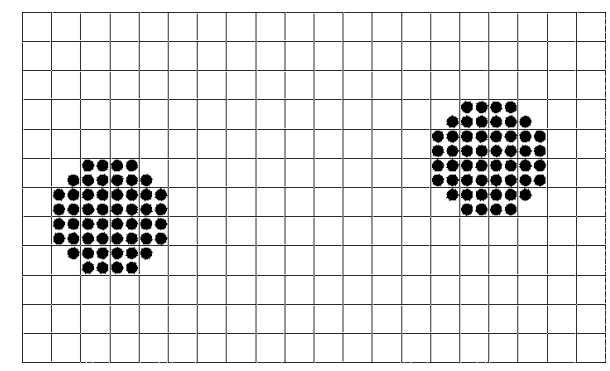

(a) Time $\mathrm{t}=0.0$

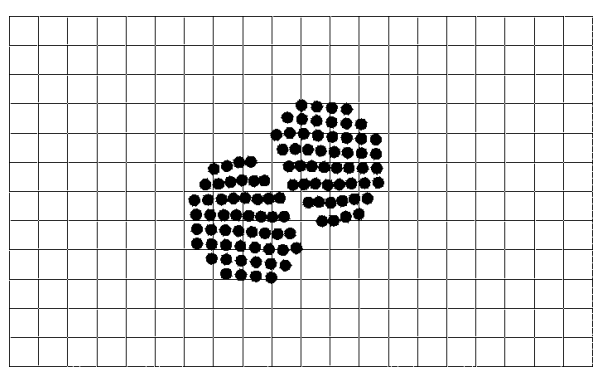

(c) Time $t=7.0$

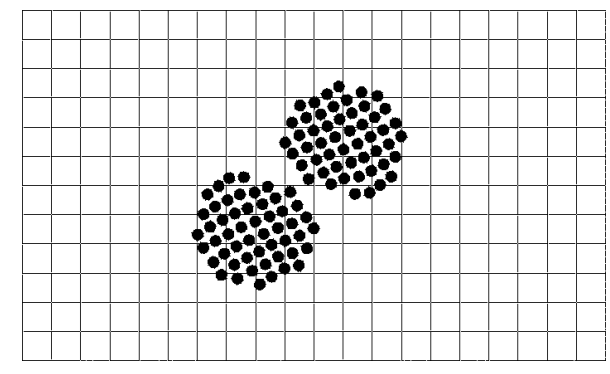

(e) Time $\mathrm{t}=9.0$

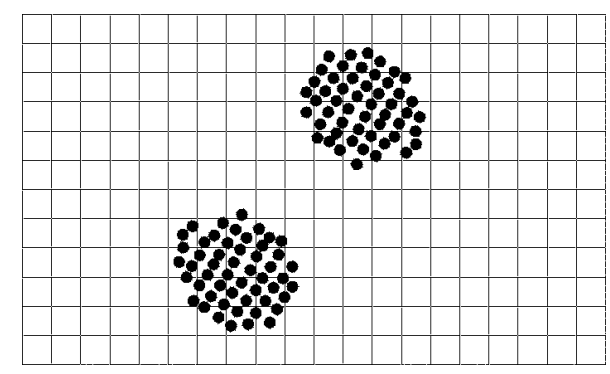

(g) Time $\mathrm{t}=12.0$

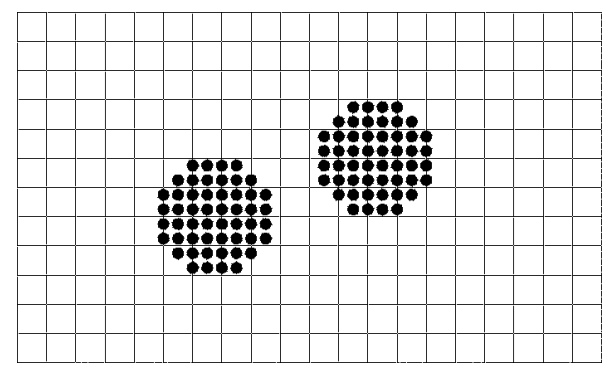

(b) Time $t=5.0$

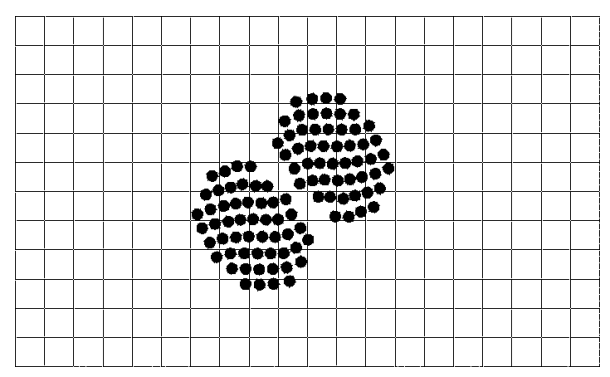

(d) Time $t=8.0$

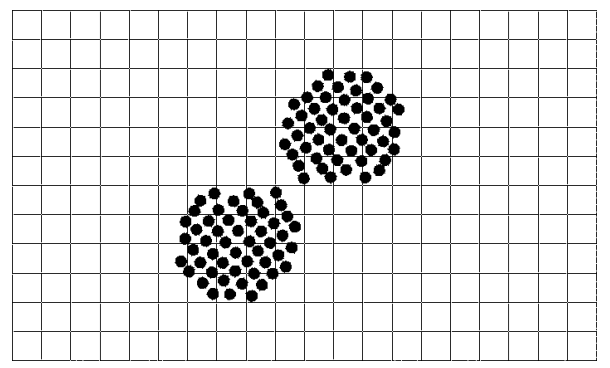

(f) Time $\mathrm{t}=10.0$

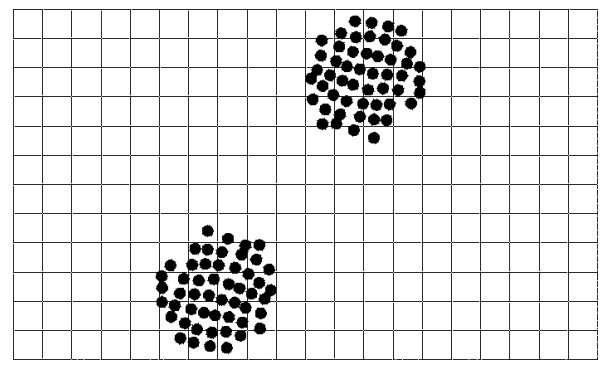

(h) Time $\mathrm{t}=14.0$

Figure 5: Skew impact of elastic cylinders. Sequence of deformed particle configurations for a consistent mass simulation. 


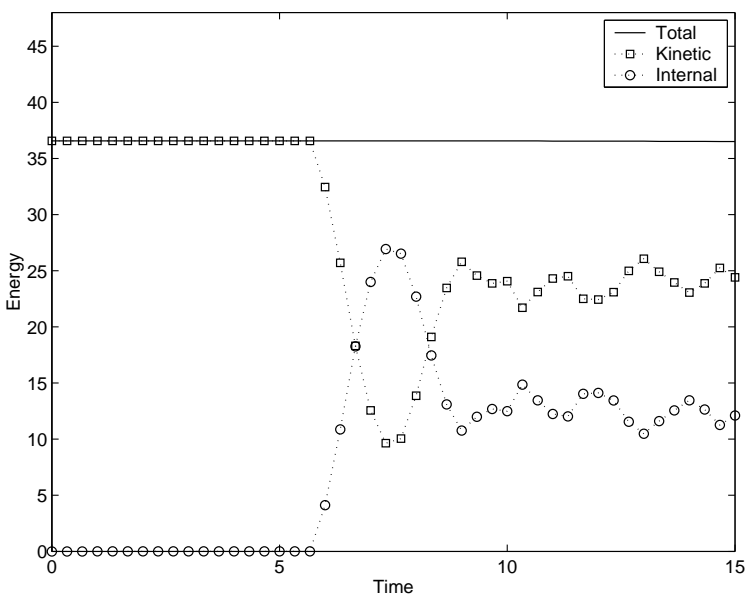

Figure 6: Skew impact of elastic cylinders. Evolution of total, kinetic and internal energies for a consistent mass calculation. The total energy is conserved.

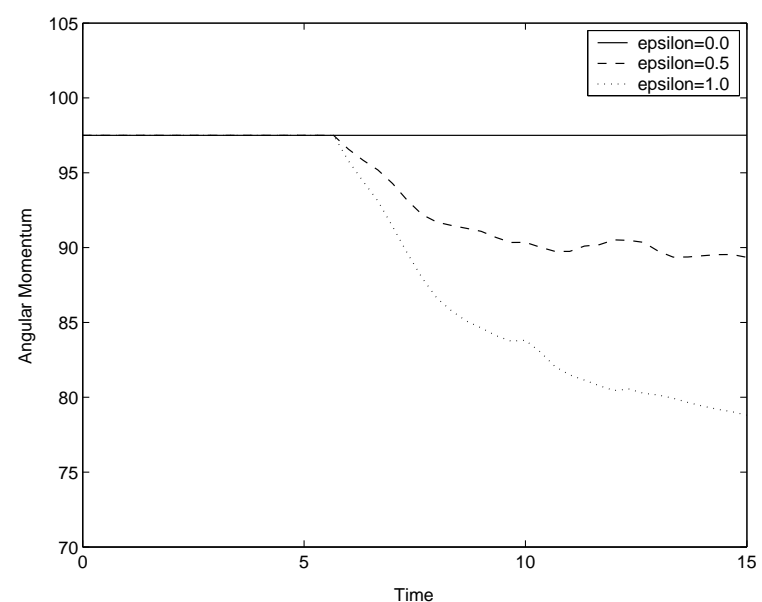

(a) Angular Momentum

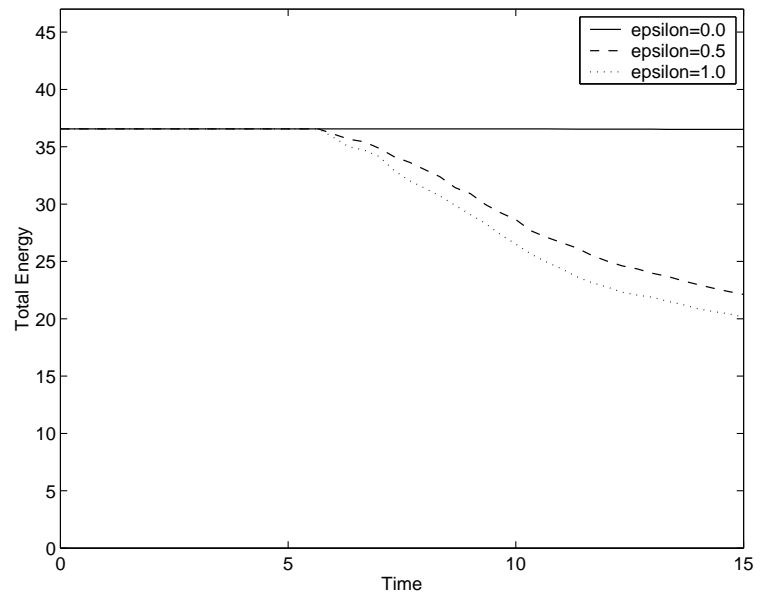

(b) Energy

Figure 7: Skew impact of elastic cylinders. (a) Evolution of angular momentum for three different choices of the parameter $\varepsilon$. Note the non-conservative behavior for $\varepsilon>0$. (b) Evolution of the total energy for three different choices of the parameter $\varepsilon$. As expected, when $\varepsilon>0$ the method is dissipative. 
momentum is conserved for the consistent mass simulation $(\varepsilon=0.0)$. The dashed line and the dotted line are plots of angular momentum versus time for simulations with $\varepsilon=0.5$ and $\varepsilon=1.0$, respectively. The skew impact of the cylinders causes them to rotate upon and after impact. The lack of angular momentum conservation is apparent in the figure when mass lumping is used. Similarly, Fig. 7(b) compares the evolution of total energy for different choices of the parameter $\varepsilon$. Again, the lack of conservation is apparent when mass lumping is used.

The evolution of the total linear momentum is not reported for any of the performed simulations. There is no need to do so. Any consistent discrete time integration scheme, in combination with the use of the weak form (6), for all values of $\varepsilon \in[0,1]$, trivially conserves linear momentum.

The Newton convergence characteristics of Algorithm MPM have not to this point been discussed. It is noted that convergence of a consistent mass calculation is more difficult to achieve than when mass lumping is used. During the course of this work, we encountered situations where the consistent mass calculation failed to converge to a solution. There are three likely contributing factors:

1. The conservative nature of the scheme makes it, by construction, harder to converge. High velocity impacts generate a full spectrum of vibrational modes that remain part of the solution. Converging under-resolved, high-frequency modes is generally difficult. Dissipative schemes exhibit better convergence characteristics [3].

2. The mass and/or stiffness matrix may be ill-conditioned.

3. Convergence difficulties have been associated with material points crossing element boundaries [43].

Typically, a consistent mass simulation takes more time to run and cannot achieve as tight a convergence tolerance as a dissipative simulation. This could perhaps be mitigated by the use of an exact Newton scheme coupled with a direct unsymmetric matrix solver, thus bypassing quasi-Newton methods and iterative solvers altogether. It may also be possible to use a pre-conditioner for the iterative solver to improve convergence, a technique not investigated in this work, but studied within an MPM context in [18].

In the numerical examples, contact is treated automatically in the MPM. Bodies do not interpenetrate because the material points move in a single-valued velocity field. This formulation necessitates the assumption of no-slip at the contact site. The contact does not affect momentum and energy conservation.

\section{Closure}

Conservation of linear and angular momenta, and conservation of energy are examined for the MPM algorithm combined with a momentum and energy conserving finite element method for the grid dynamics. When a consistent mass matrix is used, the MPM algorithm inherits the conservation properties of the grid solution. In particular, the implicit time integration scheme used for the grid dynamics is designed to reflect the continuum conservation laws 
for total energy, and linear and angular momenta. The conserving scheme applies to general hyperelastic material models and is applied to a Neo-Hookean model in the numerical examples. The examples consist of a cylinder impacting and rebounding from walls, and the skew impact of two cylinders. When a lumped mass matrix is used, total energy and angular momentum are no longer conserved. The discussion in Section 3 gives a specific expression for the error in energy and angular momentum and shows how the error arises. The numerical simulations confirm the theoretical behavior.

As mentioned above, the consistent mass formulation can be difficult to converge and perhaps pre-conditioning should be considered for the iterative solver. There are several other logical extensions of this work. First, it is of interest to develop energy and momentum consistent formulations for dynamic finite strain plasticity. Again the MPM algorithm can take advantage of developments in this area for the finite element grid dynamics [34], [35]. Another promising area is the simulation of Newtonian fluid dynamics, possibly with fluid-structure interaction. The MPM algorithm is more suited to flow calculations than Lagrangian finite element methods. Finally, it is of interest to examine frictional contact within MPM in the context of energy and momentum conserving methods. The frictional contact algorithms developed for MPM [7], [18] need modification to ensure energy consistency.

Acknowledgment: This material is based upon work supported by the National Science Foundation under Grant No. DMS-0222253. Any opinions, findings, and conclusions or recommendations expressed in this material are those of the author(s) and do not necessarily reflect the views of the National Science Foundation.

\section{References}

[1] Stuart S. Antman. Nonlinear Problems of Elasticity. Springer-Verlag, New York, 1995.

[2] F. Armero and E. Petocz. Formulation and analysis of conserving algorithms for frictionless dynamic contact/impact problems. Computer Methods in Applied Mechanics and Engineering, 158(3-4):269-300, June 1998.

[3] F. Armero and I. Romero. On the formulation of high-frequency dissipative timestepping algorithms for nonlinear dynamics, part I: Low-order methods for two model problems and nonlinear elastodynamics. Computer Methods in Applied Mechanics and Engineering, 190:2603-2649, 2001.

[4] Satish Balay, Kris Buschelman, Victor Eijkhout, William D. Gropp, Dinesh Kaushik, Matthew G. Knepley, Lois Curfman McInnes, Barry F. Smith, and Hong Zhang. PETSc users manual. Technical Report ANL-95/11 - Revision 2.1.5, Argonne National Laboratory, 2004.

[5] Satish Balay, Kris Buschelman, William D. Gropp, Dinesh Kaushik, Matthew G. Knepley, Lois Curfman McInnes, Barry F. Smith, and Hong Zhang. PETSc Web page, 2001. http://www.mcs.anl.gov/petsc. 
[6] Satish Balay, Victor Eijkhout, William D. Gropp, Lois Curfman McInnes, and Barry F. Smith. Efficient management of parallelism in object oriented numerical software libraries. In E. Arge, A. M. Bruaset, and H. P. Langtangen, editors, Modern Software Tools in Scientific Computing, pages 163-202. Birkhäuser Press, 1997.

[7] S. Bardenhagen, J. Brackbill, and D. Sulsky. The material-point method for granular materials. Computer Methods in Applied Mechanics and Engineering, 187:529-541, 2000 .

[8] Ted Belytschko, Wing Kam Liu, and Brain Moran. Nonlinear Finite Elements for Continua and Structures. John Wiley and Sons, New York, 2000.

[9] J. Bonet and P. Bhargava. The incremental flow formulation for the numerical analysis of 3-dimensional viscous deformation processes: Continuum formulation and computational aspects. Computer Methods in Applied Mechanics and Engineering, 122(12):51-68, April 1995.

[10] Javier Bonet and Richard D. Wood. Nonlinear Continuum Mechanics for Finite Element Analysis. Cambridge University Press, New York, 1997.

[11] J.U. Brackbill. On modeling angular momentum and vorticity in compressible fluid flow. Computer Physics Communications, 47:1-16, 1987.

[12] J.U. Brackbill, D. B. Kothe, and H. M. Ruppel. FLIP: A low-dissipation, particle-in-cell method for fluid flow. Computer Physics Communications, 48:25-38, 1988.

[13] J.U. Brackbill and H. M. Ruppel. FLIP: A method for adaptively zoned, particle-in-cell calculations in two dimensions. Journal of Computational Physics, 65:314-343, 1986.

[14] C. G. Broyden. A class of methods for solving nonlinear simultaneous equations. Mathematics of Computation, 19(92):577-593, October 1965.

[15] Richard L. Burden and J. Douglas Faires. Numerical Analysis. PWS-Kent Publishing Company, Boston, 4th edition, 1988.

[16] D. Burgess, D. Sulsky, and J. U. Brackbill. Mass matrix formulation of the FLIP particle-in-cell method. Journal of Computational Physics, 103(1):1-15, 1992.

[17] Philippe G. Ciarlet. Mathematical Elasticity, Volume I: Three-Dimensional Elasticity, volume 20 of Studies in Mathematics and its Applications. North-Holland, New York, 1988.

[18] S. J. Cummins and J. U. Brackbill. An implicit particle-in-cell method for granular materials. Journal of Computational Physics, 180:506-548, 2002.

[19] J. E. Dennis, Jr. and J. J. More. Quasi-newton methods, motivation and theory. SIAM Review, 19(1):46-89, January 1977.

[20] S. C. Eisenstat and H. F. Walker. Choosing the forcing terms in an inexact newton method. SIAM Journal on Scientific Computing, 17:1632, 1996. 
[21] O. Gonzalez. Exact energy-momentum conserving algorithms for general models in nonlinear elasticity. Computer Methods in Applied Mechanics and Engineering, 190:17631783, 2000.

[22] Oscar Gonzalez. Design and Analysis of Conserving Integrators for Nonlinear Hamiltonian Systems with Symmetry. PhD thesis, Department of Mechanical Engineering, Stanford University, Stanford, CA, 1996.

[23] J. E. Guilkey and J. A. Weiss. Implicit time integration for the material point method: Quantitative and algorithmic comparisons with the finite element method. International Journal for Numerical Methods in Engineering, 57:1323-1338, 2003.

[24] Morton E. Gurtin. An Introduction to Continuum Mechanics. Academic Press, New York, 1981.

[25] R.W. Hockney and J.W. Eastwood. Computer Simulation Using Particles. Adam Hilger, New York, 1988.

[26] Thomas J. R. Hughes. The Finite Element Method: Linear Static and Dynamic Finite Element Analysis. Dover Publications, New York, September 2000.

[27] Eugene Isaacson and Herbert Bishop Keller. Analysis of Numerical Methods. John Wiley and Sons, New York, 1966.

[28] C. Kane, J. E. Marsden, M. Ortiz, and M. West. Variational integrators and the newmark algorithm for conservative and dissipative mechanical systems. International Journal for Numerical Methods in Engineering, 49:1295-1325, 2000.

[29] Ann M. Kaul. Development of an Implicit Material Point Method With Application to Metals Processing. PhD thesis, Department of Mathematics and Statistics, University of New Mexico, Albuquerque, December 2000.

[30] T. A. Laursen and X. N. Meng. A new solution procedure for application of energyconserving algorithms to general constitutive models in nonlinear elastodynamics. Computer Methods in Applied Mechanics and Engineering, 190:6309-6322, 2001.

[31] N.-S. Lee and K. J. Bathe. Error indicators and adaptive remeshing in large deformation finite element analysis. Finite Elements in Analysis and Design, 16(2):99-139, May 1994.

[32] A. Lew, J. E. Marsden, M. Ortiz, and M. West. Variational time integrators. International Journal for Numerical Methods in Engineering, 60:153-212, 2004.

[33] Jerrold E. Marsden and Thomas J. R. Hughes. Mathematical Foundations of Elasticity. Dover Publications, New York, 1994.

[34] X. N. Meng and T. A. Laursen. Energy consistent algorithms for dynamic finite deformation plasticity. Computer Methods in Applied Mechanics and Engineering, 191:1639$1675,2002$. 
[35] X. N. Meng and T. A. Laursen. On energy consistency of large deformation plasticity models, with application to the design of unconditionally stable time integrators. Finite Elements in Analysis and Design, 38:949-963, 2002.

[36] L. Noels, L. Stainier, and J. P. Ponthot. An energy-momentum conserving algorithm for non-linear hypoelastic constitutive models. International Journal for Numerical Methods in Engineering, 59:83-114, 2004.

[37] J. T. Oden. Finite Elements of Nonlinear Continua. McGraw-Hill, San Francisco, 1972.

[38] R. W. Ogden. Non-Linear Elastic Deformations. Dover Publications, New York, 1997.

[39] J. C. Simo. Numerical analysis and simulation of plasticity. In P.G. Ciarlet and J. L. Lions, editors, Handbook Of Numerical Analysis, volume VI, pages 183-499. Elsevier, New York, 1998.

[40] J. C. Simo and N. Tarnow. The discrete energy-momentum method. Conserving algorithms for nonlinear elastodynamics. Zeitschrift fur Angewandte Mathematik und Physik(ZAMP), 43(5):757-792, September 1992.

[41] J.C. Simo, N. Tarnow, and K. K. Wong. Exact energy-momentum conserving algorithms and symplectic schemes for nonlinear dynamics. Computer Methods in Applied Mechanics and Engineering, 100:63-116, 1992.

[42] D. Sulsky, Z. Chen, and H. L. Schreyer. A particle method for history-dependent materials. Computer Methods in Applied Mechanics and Engineering, 118:179-196, 1994.

[43] D. Sulsky and A. Kaul. Implicit dynamics in the material-point method. Computer Methods in Applied Mechanics and Engineering, 193:1137-1170, 2004.

[44] D. Sulsky and H. L. Schreyer. Axisymmetric form of the material point method with applications to upsetting and Taylor impact problems. Computer Methods in Applied Mechanics and Engineering, 139:409-429, 1996.

[45] D. Sulsky, S.-J. Zhou, and H. L. Schreyer. Application of a particle-in-cell method to solid mechanics. Computer Physics Communications, 87:236-252, 1995.

[46] C. Truesdell. Hypoelasticity. Journal of Rational Mechanics and Analysis, 4:83-133, 1955.

[47] O. C. Zienkiewicz and R. L. Taylor. The Finite Element Method: Volume 1, The Basis. Butterworth-Heinemann, Boston, 5th edition, August 2000. 


\section{A Conservation of momentum for the Lagrangian mesh dynamics}

This appendix provides proofs for Propositions 3.5-3.6 that show conservation of total linear and angular momentum during solution of the momentum equation on the background mesh.

Proposition A.1. Assume $\left(\mathbf{F}^{e x t}\right)_{n+1 / 2}^{A}=\mathbf{0}$. Then the mesh dynamics conserve exactly the total mesh linear momentum, $\Delta \mathbf{L}^{h}=0$.

Proof. Since during the Lagrangian phase the mass matrix is held constant, the proof is simple algebra:

$$
\begin{aligned}
\frac{1}{\Delta \mathrm{t}}\left(\mathbf{L}_{n+1}^{h}-\mathbf{L}_{n}^{h}\right) & =\sum_{A=1}^{N_{\text {nodes }}}\left(\frac{1}{\Delta \mathrm{t}} \sum_{B=1}^{N_{\text {nodes }}} \widetilde{M}_{n}^{A B}\left(\mathbf{v}_{B, n+1}-\mathbf{v}_{B, n}\right)\right) \\
& =-\sum_{A=1}^{N_{\text {nodes }}}\left(\mathbf{F}^{\text {int }}\right)_{n+1 / 2}^{A} \\
& =-\sum_{A=1}^{N_{\text {nodes }}} \sum_{p t=1}^{N_{p t}}\left(\mathbf{F}_{n+1 / 2}^{p t} \mathbf{S}^{p t}\right)\left(\mathbf{F}_{n}^{p t}\right)^{T} \operatorname{grad}_{n}\left[N^{A}\left(\mathbf{x}_{n}^{p t}\right)\right] \Omega_{0} \\
& =-\sum_{p t=1}^{N_{p t}}\left(\mathbf{F}_{n+1 / 2}^{p t} \mathbf{S}^{p t}\right) \operatorname{GRAD}_{\mathbf{X}}\left[\sum_{A=1}^{N_{\text {nodes }}} N^{A}\left(\mathbf{x}_{n}^{p t}\right)\right] \Omega_{0} \\
& =-\sum_{p t=1}^{N_{p t}}\left(\mathbf{F}_{n+1 / 2}^{p t} \mathbf{S}^{p t}\right) \operatorname{GRAD}_{\mathbf{X}}[1] \Omega_{0} \\
& =\mathbf{0}
\end{aligned}
$$

which implies that $\mathbf{L}_{n+1}^{h}=\mathbf{L}_{n}^{h}$, the desired result.

Lemma A.2. There exists a linear mapping $\mathbb{E}: \mathcal{T}_{3 \times 3} \rightarrow \mathbb{R}^{3}$ such that:

1. $\mathbb{E}(\mathbf{a} \otimes \mathbf{b})=(\mathbf{a} \times \mathbf{b}) \quad \forall \mathbf{a}, \mathbf{b} \in \mathbb{R}^{3}$.

2. $\mathbb{E}(\mathbf{A})=\mathbf{0} \forall \mathbf{A} \in \mathcal{T}_{3 \times 3}^{\text {sym }}$.

Proof. $\mathbb{E}$ is the alternating tensor defined in section 2.2.4 of reference [10].

Proposition A.3. Assume $\left(\mathbf{F}^{e x t}\right)_{n+1 / 2}^{A}=\mathbf{0}$. Then the mesh dynamics conserve exactly the total mesh angular momentum, $\Delta \mathbf{J}^{h}=0$.

Proof. The presentation follows closely that of Proposition 4.1 in [3] and Proposition 2 in [21]. (See also Theorem 53.1 in [39]). The proof is algebraically tedious. As such, a number of intermediate results are useful. First, for any nodal velocity field $\mathbf{v}^{h}$, we have

$$
\sum_{A, B=1}^{N_{\text {nodes }}} \mathbf{v}_{A} \times \widetilde{M}^{A B} \mathbf{v}_{B}=\mathbf{0}
$$


since the mass matrix is symmetric and the cross product is a skew-symmetric multi-linear operator. Next, observe

$$
\begin{aligned}
\sum_{A, B=1}^{N_{\text {nodes }}}\left(\boldsymbol{\varphi}_{A, n} \times \widetilde{M^{A B}} \mathbf{v}_{B, n+1}\right. & \left.-\boldsymbol{\varphi}_{A, n+1} \times \widetilde{M}^{A B} \mathbf{v}_{B, n}\right)= \\
= & \sum_{A, B=1}^{N_{\text {nodes }}}\left(\boldsymbol{\varphi}_{A, n+1}-\mathbf{v}_{A, n+1 / 2} \Delta \mathrm{t}\right) \times \widetilde{M}^{A B} \mathbf{v}_{B, n+1} \\
= & -\sum_{A, B=1}^{N_{\text {nodes }}}\left(\boldsymbol{\varphi}_{A, n}^{h}+\mathbf{v}_{A, n+1 / 2} \Delta \mathrm{t}\right) \times \widetilde{M}^{A B} \mathbf{v}_{B, n} \quad\left(\text { by }(29)_{2}\right) \\
& -\Delta \mathrm{t} \sum_{A, B=1}^{N_{\text {nodes }}} \mathbf{v}_{A, n+1 / 2} \times \widetilde{M}^{A B}\left(\mathbf{v}_{B, n}+\mathbf{v}_{B, n+1}\right) \\
= & \left(\mathbf{J}_{n+1}^{h}-\mathbf{J}_{n}^{h}\right)-2 \Delta \mathrm{t} \sum_{A, B=1}^{N_{\text {nodes }}} \mathbf{v}_{A, n+1 / 2} \times \widetilde{M}^{A B} \mathbf{v}_{B, n+1 / 2} \\
= & \mathbf{J}_{n+1}^{h}-\mathbf{J}_{n}^{h}(\text { by }(72)) .
\end{aligned}
$$

Continuing, use this result to establish

$$
\begin{aligned}
\sum_{A, B=1}^{N_{\text {nodes }}}\left(\boldsymbol{\varphi}_{A, n+1 / 2} \times \widetilde{M}^{A B} \Delta \mathbf{v}_{B}\right)= & \frac{1}{2} \sum_{A, B=1}^{N_{\text {nodes }}}\left(\left(\boldsymbol{\varphi}_{A, n}+\boldsymbol{\varphi}_{A, n+1}\right) \times \widetilde{M}^{A B}\left(\mathbf{v}_{B, n+1}-\mathbf{v}_{B, n}\right)\right) \\
= & \frac{1}{2}\left(\mathbf{J}_{n+1}^{h}-\mathbf{J}_{n}^{h}\right) \\
& +\frac{1}{2} \sum_{A, B=1}^{N_{\text {nodes }}}\left(\boldsymbol{\varphi}_{A, n} \times \widetilde{M}^{A B} \mathbf{v}_{B, n+1}-\boldsymbol{\varphi}_{A, n+1} \times \widetilde{M}^{A B} \mathbf{v}_{B, n}\right) \\
= & \frac{1}{2}\left(\mathbf{J}_{n+1}^{h}-\mathbf{J}_{n}^{h}\right)+\frac{1}{2}\left(\mathbf{J}_{n+1}^{h}-\mathbf{J}_{n}^{h}\right) \quad(\text { by }(73)) \\
= & \mathbf{J}_{n+1}^{h}-\mathbf{J}_{n}^{h} .
\end{aligned}
$$


The moment of the internal force is

$$
\begin{aligned}
\sum_{A=1}^{N_{\text {nodes }}} \boldsymbol{\varphi}_{A, n+1 / 2} \times \mathbf{F}_{n+1 / 2}^{\text {int }} & =\sum_{A=1}^{N_{\text {nodes }}} \boldsymbol{\varphi}_{A, n+1 / 2} \times \sum_{p t=1}^{N_{p t}}\left(\mathbf{F}_{n+1 / 2}^{p t} \mathbf{S}^{p t}\right) \underbrace{\left(\mathbf{F}_{n}^{p t}\right)^{T} \operatorname{grad}_{n}\left[N^{A}\left(\mathbf{x}_{n}^{p t}\right)\right]}_{\operatorname{GRAD}_{\mathbf{X}}\left[N^{A}\left(\mathbf{x}_{n}^{p t}\right)\right]} \Omega_{0}^{p t} \\
& =\sum_{A=1}^{N_{\text {nodes }}} \sum_{p t=1}^{N_{p t}} \boldsymbol{\varphi}_{A, n+1 / 2} \times\left[\mathbf{F}_{n+1 / 2}^{p t} \mathbf{S}^{p t} \operatorname{GRAD}_{\mathbf{X}}\left[N^{A}\left(\mathbf{x}_{n}^{p t}\right)\right]\right] \Omega_{0}^{p t} \\
& =\sum_{A=1}^{N_{\text {nodes }}} \sum_{p t=1}^{N_{p t}} \mathbb{E}\left[\boldsymbol{\varphi}_{A, n+1 / 2} \otimes\left(\mathbf{F}_{n+1 / 2}^{p t} \mathbf{S}^{p t} \operatorname{GRAD}_{\mathbf{X}}\left[N^{A}\left(\mathbf{x}_{n}^{p t}\right)\right]\right)\right] \Omega_{0}^{p t} \\
& =\sum_{A=1}^{N_{\text {nodes }}} \sum_{p t=1}^{N_{p t}} \mathbb{E}\left[\left(\boldsymbol{\varphi}_{A, n+1 / 2} \otimes \mathrm{GRAD}_{\mathbf{X}}\left[N^{A}\left(\mathbf{x}_{n}^{p t}\right)\right]\right) \mathbf{S}^{p t}\left(\mathbf{F}_{n+1 / 2}^{p t}\right)^{T}\right] \Omega_{0}^{p t} \\
& =\sum_{p t=1}^{N_{p t}} \mathbb{E}\left[\sum_{A=1}^{N_{n o d e s}}\left(\boldsymbol{\varphi}_{A, n+1 / 2} \otimes \operatorname{GRAD}_{\mathbf{X}}\left[N^{A}\left(\mathbf{x}_{n}^{p t}\right)\right]\right) \mathbf{S}^{p t}\left(\mathbf{F}_{n+1 / 2}^{p t}\right)^{T}\right] \Omega_{0}^{p t} \\
& =\sum_{p t=1}^{N_{p t}} \mathbb{E}\left[\mathbf{F}_{n+1 / 2}^{p t} \mathbf{S}^{p t}\left(\mathbf{F}_{n+1 / 2}^{p t}\right)^{T}\right] \Omega_{0}^{p t} \\
& =\mathbf{0 .}
\end{aligned}
$$

Notice that the symmetry of $\mathbf{S}^{p t}$ has been used above. Finally, consideration of equation (29) 1 yields

$$
\begin{aligned}
\mathbf{0} & =\sum_{A=1}^{N_{\text {Nodes }}} \boldsymbol{\varphi}_{A, n+1 / 2} \times\left[\frac{1}{\Delta \mathrm{t}} \sum_{B=1}^{N_{\text {Nodes }}} \widetilde{M}_{n}^{A B}\left(\mathbf{v}_{B, n+1}-\mathbf{v}_{B, n}\right)+\left(\mathbf{F}^{i n t}\right)_{n+1 / 2}^{A}\right] \\
& =\sum_{A=1}^{N_{\text {Nodes }}} \boldsymbol{\varphi}_{A, n+1 / 2} \times\left[\frac{1}{\Delta \mathrm{t}} \sum_{B=1}^{N_{\text {Nodes }}} \widetilde{M}_{n}^{A B} \Delta \mathbf{v}_{B}+\left(\mathbf{F}^{\text {int }}\right)_{n+1 / 2}^{A}\right] \\
& =\frac{1}{\Delta \mathrm{t}}\left(\mathbf{J}_{n+1}^{h}-\mathbf{J}_{n}^{h}\right) \quad \text { by }((74) \text { and }(75)) .
\end{aligned}
$$

Since $\Delta \mathrm{t}>0, \mathbf{J}_{n+1}^{h}=\mathbf{J}_{n}^{h}$, which is the desired result. 
